\title{
THE INTEGRAL COHOMOLOGY RINGS OF GROUPS OF ORDER $p^{3}$
}

\author{
BY \\ GENE LEWIS( ${ }^{(1)}$
}

0. Introduction. Most of the papers in cohomology of groups have been theoretical [1], [3]-[6], [8], [9], [13], [14], rather than computational. The following list describes all the (published and unpublished) computations I am aware of. Atiyah $[1$, p. 60] calculated the integral (cohomology) ring of the quaternion group $Q$ of order 8. Wall [16] obtained the additive structure of split cyclic-by-cyclic extension. He could not get the ring structure. Cartan-Eilenberg [3, Chapter XII, §7] calculated the cohomology of a cyclic group with any coefficients, and gave an explicit diagonal map. Evens [6] wrote down the cohomology ring of the dihedral group $D$ of order 8 . Unfortunately, he did not publish the details of his calculations. Nakaoka [9] calculated the homology ring of symmetric groups with coefficients $Z_{p}$ and in [10, p. 52] found the cohomology ring of $\Sigma_{4}$, coefficients $Z_{2}$. Cardenas [2] calculated the ring of $\Sigma_{p^{2}}$, coefficients $Z_{p}$. Tate [15] got the integral homology ring of any finitely generated abelian group.

Finally, Norman Hamilton and Arnold Shapiro got the integral cohomology ring of $Z_{p}>Z_{p}$, but neglected to write it down.

In this paper, I will find the integral cohomology rings of groups of order $p^{3}$, except for $Q$ and $D$. I merely sketch the abelian cases, as they are trivial.

\section{Review of basic facts.}

1.1. We recall some basic results on change of groups, transfer, and spectral sequences. Throughout this paper, all $G$-modules are left $G$-modules, all exact sequences of $G$-modules are assumed $Z$-split, and all groups are finite (in many places, this last assumption is superfluous). Let $G, G^{\prime}$ be groups, $A, A^{\prime}$ be $G$ (resp. $G^{\prime}$ )-modules.

Definition. A change of groups $\phi:(G, A) \rightarrow\left(G^{\prime}, A^{\prime}\right)$ is a pair $(\varphi, \alpha)$ such that $\varphi: G^{\prime} \rightarrow G$ is a morphism, and $\alpha: A_{(\varphi)} \rightarrow A^{\prime}$ is a $G^{\prime}$-morphism.

A change of groups $\phi$ induces a morphism $\phi^{*}: H^{n}(G, A) \rightarrow H^{n}\left(G^{\prime}, A^{\prime}\right), n \geqq 0$. $\phi^{*}$ is obtained as follows: Let $X$ be a $G$-projective resolution of $Z, Y$ a $G^{\prime}$-projective resolution of $Z$. The comparison theorem gives a homotopy class of chain maps $Y \stackrel{f}{\rightarrow} X_{(\varphi)}$. For $\xi \in H^{n}(G, A)$, with representative cocycle $h: X_{n} \rightarrow A$, we let $\phi^{*}(h)$ be the image of

$$
\begin{aligned}
h \in \operatorname{Hom}_{G}(X, A) \stackrel{\text { incl }}{\longrightarrow} \operatorname{Hom}_{G^{\prime}}\left(X_{(\varphi)}, A_{(\varphi)}\right) \\
\stackrel{f^{*}}{\longrightarrow} \operatorname{Hom}_{G^{\prime}}\left(Y, A_{(\varphi)}\right) \stackrel{\alpha_{*}}{\longrightarrow} \operatorname{Hom}_{G^{\prime}}\left(Y, A^{\prime}\right)
\end{aligned}
$$

Received by the editors October 21, 1966.

(1) This research was done when the author was an NSF Graduate Fellow. 
under the composition $\alpha_{*} \circ f^{*} \circ \iota$. Let $\phi^{*}(\xi) \in H^{n}\left(G, A^{\prime}\right)$ be the cohomology class of $\phi^{*}(h)$. It is easy to see that $\phi^{*}$ is independent of the choice of $X$ and $Y$. If $(G, A) \stackrel{\oplus}{\rightarrow}\left(G^{\prime}, A^{\prime}\right) \stackrel{\Downarrow}{\longrightarrow}\left(G^{\prime \prime}, A^{\prime \prime}\right)$ are changes of groups, then if we define $(G, A)$ $\stackrel{\psi \circ \phi}{\longrightarrow}\left(G^{\prime \prime}, A^{\prime \prime}\right)$ in the obvious way, it is a change of groups, and $(\psi \circ \phi)^{*}=\psi^{*} \circ \phi^{*}$. In particular, if $\lambda: G \rightarrow G$ is an automorphism, we get a change of groups $\lambda:(G, A) \rightarrow\left(G, A_{(\lambda)}\right)$ given by $\lambda: G \rightarrow G$ and identity $A_{(\lambda)} \rightarrow A_{(\lambda)}$. In terms of standard cocycles, this is given by $[h] \in H^{n}(G, A) \rightarrow[h \circ f] \in H^{n}\left(G, A_{(\lambda)}\right)$, where $f: B G \rightarrow B G_{(\lambda)}$ is $f\left(\left[g_{1}, \ldots, g_{n}\right]\right)=\left[\lambda\left(g_{1}\right), \ldots, \lambda\left(g_{n}\right)\right], f$ a $G$-morphism. For $A=Z$, trivial action, this gives a right action of Aut $(G)$ on $H^{*}(G, Z)$, which evidently preserves products. If $A=G$-module, $H \subset G$, subgroup, $\sigma \in N_{G}(H)$, then $\sigma$ induces $\lambda_{\sigma}: H \rightarrow H, \quad \lambda_{\sigma}(h)=\sigma^{-1} h \sigma=h^{\sigma}$. Let $X=G$-projective resolution of $Z$. Then $(H, A) \stackrel{\lambda_{\sigma}}{\rightarrow}(H, A)$ given by $\lambda_{\sigma}: H \rightarrow H, A \stackrel{\sigma}{\leftarrow} A$ is a change of groups. The corresponding $f: X \rightarrow X_{\left(\lambda_{\sigma}\right)}$ is multiplication by $\sigma^{-1}$. Thus $\lambda_{\sigma}^{*}$ is got as follows: For $[h] \in H^{n}(H, A), h: X_{n} \rightarrow A$, cocycle, form $\sigma h$, where $\sigma h(x)=\sigma \cdot h\left(\sigma^{-1} x\right)$. Then $\lambda_{\sigma}^{*}[h]=[\sigma h]$. As $\sigma \rightarrow \lambda_{\sigma}$ is an antimorphism of $G \rightarrow$ Aut $G,[h] \rightarrow \lambda_{\sigma}^{*}[h]$ gives a left action of $N_{G}(H)$ on $H^{*}(H, A)$. In terms of standard $H$-cocycles,

$$
\lambda_{\sigma}^{*}[h]\left(\left[h_{1}, \ldots, h_{n}\right]\right)=\sigma \cdot h\left(\left[h_{1}^{\sigma}, \ldots, h_{n}^{\sigma}\right]\right)
$$

$\left[8\right.$, p. 117]. For we now use $f: B H \rightarrow B H_{\left(\lambda_{\sigma}\right)}$ such that

$$
f\left(\left[h_{1}, \ldots, h_{n}\right]\right)=\left[\lambda_{\sigma}\left(h_{1}\right), \ldots, \lambda_{\sigma}\left(h_{n}\right)\right]
$$

and then compose with $A \stackrel{\sigma}{\leftarrow} A$.

1.2. As Cor commutes with coboundaries, the following commutes

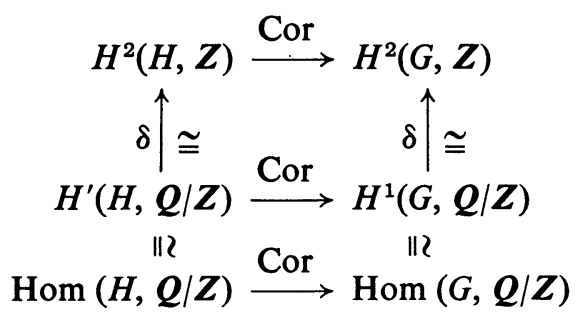

Let $G=\bigcup \sigma_{i} H$, disjoint, $g \sigma_{i}=\sigma_{\pi(i)} h(i, g)$. Given $f: H \rightarrow \boldsymbol{Q} / Z$, morphism, we have: Cor $f: g \rightarrow \sum_{i} f(h(i, g))$.

The proof is an easy calculation, which we leave to the reader.

1.3. If $1 \rightarrow H \rightarrow G \rightarrow K \rightarrow 1$ is a group extension, $A$ a $G$-module, then there is a spectral sequence $E_{2} \Rightarrow H^{*}(G, A)$ [8], where $E_{2}^{i, j} \cong H^{i}\left(K, H^{j}(H, A)\right)$. In case $A=R$ =a $G$-ring, the isomorphism $E_{2} \cong{ }^{\varphi} H^{*}\left(K, H^{*}(H, R)\right)$ is an isomorphism of bigraded rings, up to a sign: if $\alpha \in H^{i}\left(K, H^{j}(H, R)\right), \beta \in H^{k}\left(K, H^{l}(H, R)\right)$, then $\varphi(\alpha \beta)$ $=(-1)^{j k} \varphi(\alpha) \varphi(\beta)$. A check on this is provided by noting that in $H^{*}\left(K, H^{*}(H, R)\right)$ we have

(1) $\alpha \beta=(-1)^{i k+j l} \beta \alpha$, whereas in $E_{2}$ we need

(2) $\varphi(\alpha) \varphi(\beta)=(-1)^{(i+j)(k+l)} \varphi(\beta) \varphi(\alpha)$. 
With the sign $(-1)^{j k}, \varphi$ transforms (1) into (2). Recall also the commutative diagrams $[4$, p. 227]

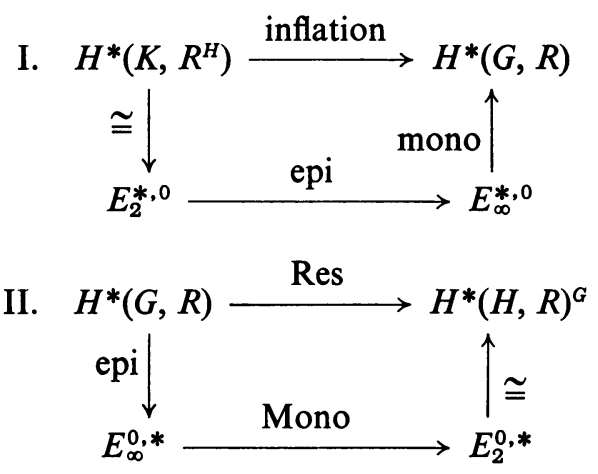

COROLlaRY $(\mathrm{I}) \Rightarrow$ : in a split extension, since $\operatorname{Inf}$ is then mono, we have $E_{2}^{*, 0} \cong E_{\infty}^{*, 0}$.

COROLLARY (II) $\Rightarrow:$ universal cycles of $E_{2}^{0, *}$ are in the image of the restriction.

Remark. It can be shown that from the $E_{2}$ term on, the spectral sequence (including the multiplication and differentials) is an invariant of the extension.

2. The restriction-corestriction sequences. Suppose $1 \rightarrow H \rightarrow G \rightarrow K \rightarrow 1$ is a group extension, such that $K$ is cyclic of order $s$, with generator $k$. The exact sequence

$$
0 \longleftarrow Z \stackrel{\varepsilon}{\longleftarrow} Z K \stackrel{k-1}{\longleftarrow} Z K \stackrel{\theta}{\longleftarrow} Z \longleftarrow 0
$$

is an exact sequence of $G$-modules, via inflation. Let $X=\operatorname{Ker} \varepsilon=\operatorname{Im}(k-1)$. We get:

(2) $0 \longleftarrow Z \stackrel{\varepsilon}{\longleftarrow} Z K \stackrel{\mu}{\longleftarrow} X \longleftarrow 0$. Apply the long exact sequence of

(2) $\quad 0 \longleftarrow X \longleftarrow Z K \stackrel{\theta}{\longleftarrow} Z \longleftarrow 0$ cohomology. This gives:

$$
\begin{aligned}
\cdots \longrightarrow H^{k-1}(G, Z) \stackrel{\delta}{\longrightarrow} H^{k}(G, X) \\
\stackrel{\mu_{*}}{\longrightarrow} H^{k}(G, Z K) \stackrel{\varepsilon_{*}}{\longrightarrow} H^{k}(G, Z) \stackrel{\delta}{\longrightarrow} \cdots
\end{aligned}
$$

$$
\begin{aligned}
\cdots \longrightarrow H^{k-1}(G, X) \stackrel{\delta}{\longrightarrow} H^{k}(G, Z) \\
\stackrel{\theta_{*}}{\longrightarrow} H^{k}(G, Z K) \stackrel{\varepsilon_{*}}{\longrightarrow} H^{k}(G, X) \stackrel{\delta}{\longrightarrow} \cdots .
\end{aligned}
$$

By Shapiro's Lemma, [3, p. 196], $H^{k}(H, Z) \stackrel{\omega}{\rightarrow} H^{k}(G, Z K)$ is an isomorphism. If $X=G$-projective resolution of $Z, \omega$ arises from the associativity

$$
\omega: \operatorname{Hom}_{H}\left(Z G \otimes_{G} X, Z\right) \cong \operatorname{Hom}_{G}\left(X, \operatorname{Hom}_{H}(Z G, Z)\right) .
$$


We check rapidly that Cor $=\varepsilon_{*} \circ \omega$, Res $=\omega^{-1} \circ \theta_{*}$. Also, the action $k: H^{*}(H, Z)$ $\rightarrow H^{*}(H, Z)$ is carried by $\omega$ into the map $k_{*}: H^{*}(G, Z K) \rightarrow H^{*}(G, Z K)$, induced by $k: Z K \rightarrow Z K$. Thus:

Proposition 2.1. Given $1 \rightarrow H \rightarrow G \rightarrow K \rightarrow 1 \ni K=\{k\}$, cyclic, we get exact sequences

$$
\begin{aligned}
\cdots \longrightarrow H^{k-1}(G, Z) \stackrel{\delta}{\stackrel{\text { Cor }}{\longrightarrow}} H^{k}(G, X) \stackrel{\bar{\mu}_{*}}{\longrightarrow} H^{k}(G, Z) \stackrel{\delta}{\longrightarrow} H^{k+1}(G, X) \longrightarrow \cdots
\end{aligned}
$$

$$
\begin{aligned}
\cdots \longrightarrow H^{k-1}(G, X) \stackrel{\delta}{\stackrel{\bar{\nu}_{*}}{\longrightarrow}} H^{k}(G, Z) \stackrel{\text { Res }}{\longrightarrow} H^{*}(G, X) \stackrel{\delta}{\longrightarrow} H^{k+1}(G, Z) .
\end{aligned}
$$

Here $\bar{\mu}_{*} \circ \bar{\nu}_{*}=k-1: H^{k}(H, Z) \rightarrow H^{k}(H, Z)$.

REMARK 2.0. We could have used Tate cohomology and obtained the same result.

REMARK 2.1. If $A$ is any module, then as $0 \leftarrow Z \leftarrow Z K \leftarrow Z K \leftarrow Z \leftarrow 0$ is $Z$-split, hence so is

$$
0 \rightarrow \operatorname{Hom}(Z, A) \rightarrow \operatorname{Hom}(Z K, A) \rightarrow \operatorname{Hom}(Z K, A) \rightarrow \operatorname{Hom}(Z, A) \rightarrow 0 \text {. }
$$

Therefore, this is exact $A \cong \operatorname{Hom}(Z, A)$, and if $X, \bar{X}=$ image-kernel, we get sequences (4), (4)' with $A$ in place of $Z$.

REMARK 2.2. We can get several puny results directly from (4) and (4)'.

(1) If $G$ is a $p$-group, and $H^{2 n}(G, Z)=0, H^{2 n+1}(G, Z)=0$, then $G=\{1\}$. For let $H \triangleleft G$, of index $p$. As $k-1=\bar{\mu}_{*} \circ \bar{\nu}_{*}: H^{k}(H, Z) \rightarrow H^{k}(H, Z)$ is an isomorphism, for $k=2 n, 2 n+1$, we see that $k$ has no fixed points. But $\{k\}, H^{k}(H, Z)$ are $p$-groups. Therefore $H^{k}(H, Z)=0, k=2 n, 2 n+1$, implies that $H=\{1\}$. By induction this implies that $G=Z_{p}$, a contradiction. Hence $G=\{1\}$.

(2) If $1 \triangleleft H_{1} \triangleleft H_{2} \triangleleft \cdots \triangleleft H_{r}=G$ such that $H_{i} / H_{i-1}$ cyclic, then $\left|H^{3}(G, Z)\right|$ $\leqq \prod_{i=1}^{r-1}\left|H_{i} /\left[H_{i}, H_{i}\right]\right|$. There is an alternative treatment of these sequences. Let $\bar{X}=$ a complete resolution for $\boldsymbol{Z}$,

$$
M=\{0 \leftarrow Z K \leftarrow Z K \leftarrow 0\}, \quad M^{*}=\operatorname{Hom}(M, Z) .
$$

The bicomplex $\operatorname{Hom}_{G}\left(X, M^{*}\right)$ gives rise to two spectral sequences. If

$$
T=H^{*}\left(\operatorname{Hom}_{G}\left(X, M^{*}\right)\right),
$$

we find easily the sequences:

$$
\begin{aligned}
\cdots \longrightarrow \hat{H}^{i-1}(G, Z) & \stackrel{\cup \alpha}{\longrightarrow} \hat{H}^{i+1}(G, Z) \\
& \stackrel{\rho}{\longrightarrow} T^{i+1} \stackrel{\tau}{\longrightarrow} \hat{H}^{i}(G, Z) \stackrel{\cup \alpha}{\longrightarrow} \hat{H}^{i+2}(G, Z)
\end{aligned}
$$

$$
0 \longrightarrow \hat{H}^{i}(H, Z)_{K} \stackrel{\mu}{\longrightarrow} T^{i+1} \stackrel{\varepsilon}{\longrightarrow} \hat{H}^{i+1}(G, Z)^{K} \longrightarrow 0
$$


where $\varepsilon \circ \rho=$ Res, $\tau \circ \mu=$ Cor. Here $\alpha=\inf \chi, \chi \in H^{2}(K, Z)$, a maximal generator. Again, this holds for any module in place of $\boldsymbol{Z}$.

\section{Multiply periodic resolutions.}

3.1. Definition. $G$ is periodic if and only if there is a natural isomorphism of functors, $\hat{H}^{0}(G, \cdot) \approx \hat{H}^{n}(G, \cdot)$, for some $n>0$. The least such $n$ is the period of $G$. Call it $n(G)$.

It can be shown that $n(G)$ is always even [3, Chapter XII, $\S 11$ ].

THEOREM 3.0 (ARTIN-TATE). $G$ is periodic if and only if

$$
\begin{aligned}
G & =\text { cyclic, } p \text { odd, } \\
& =\text { cyclic or generalized quaternion, } p=2 .
\end{aligned}
$$

Proof. See [3, Chapter XII, §11].

COROLlaRY. If $G$ is periodic, then so is any subgroup. Thus $Z_{p} \oplus Z_{p} \notin G$, for any $p$.

The least $n$ such that $\hat{H}^{0}(G, \cdot)_{p} \approx \hat{H}^{n}(G, \cdot)_{p}$ is called the p-period of $G$, denoted by $n_{p}(G) . n_{p}(G)<\infty$ if and only if $G_{p}$ is cyclic, $p$ odd, or cyclic or generalized quaternion, $p=2$, [3, Example 11, p. 265], $n(G)=1$. c.m. $\left\{n_{p}(G): p|| G \mid\right\}$.

THEOREM 3.1 (SWAN). If $G$ is p-periodic, then

$$
\begin{aligned}
n_{p}(G) & =2\left|N_{G}\left(G_{p}\right) / C_{G}\left(G_{p}\right)\right|, \quad p \text { odd }, \\
& =2, \quad \text { if } G_{2} \text { cyclic, } \\
& =4, \quad \text { if } G_{2} \text { generalized quaternion. }
\end{aligned}
$$

Proof. [12].

Definition. $G$ is spheriodic if and only if $G$ acts freely on $S^{n}$, some $n$.

Definition. $G$ is uniodic if and only if $G$ has a fix-point free unitary representation.

It is easy to see that uniodic implies spheriodic implies periodic.

THEOREM 3.2 (MILNOR). If $G$ is spheriodic, then every involution lies in the center.

Proof [Amer. J. Math. 79 (1957), 623-630]. As $Z_{2} \oplus Z_{2} \notin G$, there is at most one involution in $G$.

EXAMPLE. $\quad \Sigma_{3}$ is periodic of period 4 , but not spheriodic.

LEMMA 3.3. Let $1 \rightarrow H \rightarrow G \stackrel{\pi}{\rightarrow} K \rightarrow 1$ be a group extension. If $M$ is an $H$ projective G-module, $N$ a K-projective module, then $M \otimes N$, with action $g(m \otimes n)$ $=g m \otimes \pi(g) n$, is G-projective.

Proof. See [4, p. 231]. The proof given there for the split case works in general.

Definition. If $C, C^{\prime}$ are chain complexes, a morphism $f: C \rightarrow C^{\prime}$ is called a chain map of degree $r$ if (1) $f: C_{n} \rightarrow C_{n+r}$ for all $n$, (2) $f \partial=(-1)^{r} \partial f$. 
LEMMA 3.4. Let $X$ be a complete resolution of $Z$, and suppose $f: X \rightarrow X$ is a chain map of degree $-n$. Then there exists $\eta \in H^{n}(G, Z)$ such that, for all $G$-modules $A$, the map $f^{*}: \hat{H}^{i}(G, A) \rightarrow \hat{H}^{i+n}(G, A)$ is just cup-product with $\eta$.

Proof. Let $\varepsilon: X_{0} \rightarrow Z$ be the augmentation. Then $\varepsilon \circ f_{n}: X_{n} \rightarrow Z$ is an $n$-cocycle, representing a class $\eta \in H^{n}(G, Z)$. The verification that $f^{*}=\eta \cup$ is routine, and we omit details.

Definition. A finite complex $C$ is $n$-spherical if and only if $C$ has the homology of an $n$-sphere. Thus,

is exact.

$$
C=\left\{0 \leftarrow Z \leftarrow C_{0} \leftarrow \cdots \leftarrow C_{n} \leftarrow Z \leftarrow 0\right\}
$$

Lemma 3.5. If $H \triangleleft G$, and $H$ possesses a spherical projective complex, then there exists a spherical G-complex which is $H$-projective.

Proof. Let $r=|G: H|, C=$ an $n$-spherical projective $H$-complex. Let $\dot{C}$ be the augmented complex of $C$, with degrees raised by 1 . Form $\dot{C}^{r}=\dot{C} \otimes \cdots \otimes \dot{C}$ ( $r$ times). Let $C^{r}=$ the deaugmented complex of $\dot{C}^{r}$, with degrees lowered by 1 . Künneth $\Rightarrow C^{r}$ is $(n r+r-1)$-spherical.

As shown in $\left[4\right.$, p. 231], $C^{r}$ is a $\Sigma_{r} 2 H$-complex, where $\Sigma_{r}$ acts by permuting factors, and $H^{r}$ acts pointwise. A choice of left coset representatives for $H$ in $G$ yields a monomorphism $\Phi: G \rightarrow \Sigma_{r} 2 H$. Make $C^{r}$ a $G$-module via pullback by $\Phi$. This $G$-module structure is independent of the choice of coset representatives [4]. $H \triangleleft G$ implies that $\Phi(H) \subset H^{r}$. Lemma 3.3, iterated, implies that $C^{r}$ is $H^{r}$ projective, hence $H$ is projective under $\Phi$. Q.E.D.

Suppose now that $H$ and $G / H$ are periodic. By [14], [7] we know that $H$ and $G / H$ possess spherical projective complexes, $C$ and $D$. By Lemma 3.5, we may suppose that $C$ is a $G$-complex.

Let

$$
C=\left\{0 \leftarrow Z \leftarrow C_{0} \leftarrow \cdots \leftarrow C_{b} \leftarrow Z \leftarrow 0\right\} .
$$

Splicing yields periodic

$$
D=\left\{0 \leftarrow Z \leftarrow D_{0} \leftarrow \cdots \leftarrow D_{a} \leftarrow Z \leftarrow 0\right\}
$$

resolutions $X, Y$ of $Z, X$ an $H$-projective $G$-resolution, $Y$ a $G / H$-projective resolution. Let $f: X \rightarrow X, g: Y \rightarrow Y$ be the chain maps of degree $-(b+1),-(a+1)$, respectively, given by

$$
f: X_{i+b+1} \stackrel{\text { identity }}{\longrightarrow} X_{i}, \quad g: Y_{i+a+1} \stackrel{\text { identity }}{\longrightarrow} Y_{i} .
$$

(Recall that $a+1, b+1$ are necessarily even.) Form $W=Y \otimes X$. This is doubly periodic, and by Lemma 3.3, is a $G$-projective resolution of $Z$. If $\varepsilon: W \rightarrow Z$ is the augmentation, then $\varepsilon \circ(g \otimes 1), \varepsilon \circ(1 \otimes f)$ are cocycles.

Set $\eta_{1}=[\varepsilon \circ(g \otimes 1)], \eta_{2}=[\varepsilon \circ(1 \otimes f)], \eta_{1} \in H^{a+1}(G, Z)$ and $\eta_{2} \in H^{b+1}(G, Z)$. 
By Lemma 3.4, if $\varphi: W \rightarrow Z$ is a cocycle, then $\eta_{1} \cup[\varphi]=[\varphi \circ(g \times 1)]$. Thus, to multiply $[\varphi]$ by $\eta_{1}$ one translates $\varphi a+1$ units east. Similarly for $\eta_{2}$.

By iteration of the above procedure, one proves:

THEOREM 3.6. If $1 \triangleleft H_{1} \triangleleft H_{2} \triangleleft \cdots \triangleleft H_{r}=G$ is a chain of subgroups, each normal in $G$, and such that $H_{i+1} / H_{i}$ is periodic, then $G$ possesses an $(r+1)$-ply periodic projective resolution of $Z$.

REMARK. One might hope that the cohomology classes corresponding to the perpendicular directions in a multiply periodic resolution would be algebraically independent in some sense. This seems incorrect. Thus if $1 \rightarrow Z_{4} \rightarrow Q \rightarrow Z_{2} \rightarrow 1$, $Q=$ quaternion group, let $\eta=$ inflation of the maximal generator of $Z_{2} \cdot \eta$ corresponds to a translation in the obvious doubly periodic resolution. But $\eta^{2}=0$ (see $\S 4)$. Again, for groups of type II, we will see that the inflations $\eta_{1}, \eta_{2}$ of $\chi_{A}, \chi_{B}$ $\in H^{2}(G / Z(G), Z)$ satisfy $\eta_{1} \eta_{2}^{p}=\eta_{2} \eta_{1}^{p}$. But $\eta_{1}, \eta_{2}$ correspond to mutually perpendicular directions in a triply periodic resolution of $G$.

3.2. We now look at the special case $G=\left(x, y: x^{m}=y^{n}=1, x y=y x\right)$ such that $n \mid m$. Set $\Lambda=Z G$. The obvious doubly periodic resolution here is $W=\sum_{i, j \geqq 0} \Lambda a_{i, j}$ (direct). Let $a_{i, j}=0$ if $i<0$ or $j<0$. The boundary is

$$
\begin{aligned}
\partial a_{i, j} & =T_{y} a_{i-1, j}-T_{x} a_{i, j-1} & & i, j \text { odd, } \\
& =N_{y} a_{i-1, j}+N_{x} a_{i, j-1} & & i, j \text { even, } \\
& =T_{y} a_{i-1, j}-N_{x} a_{i, j-1} & & i \text { odd, } j \text { even, } \\
& =N_{y} a_{i-1, j}+T_{x} a_{i, j-1} & & i \text { even, } j \text { odd, } \\
& & & \text { with } T_{x}=x-1, \quad N_{x}=\sum_{i=0}^{m-1} x^{m}, \text { and so on. }
\end{aligned}
$$

The periodicity chain maps $\eta_{1}, \eta_{2}: W \rightarrow W$ are of degree $-2 . \eta_{1}: a_{i, j} \rightarrow a_{i-2, j}$, $\eta_{2}: a_{i, j} \rightarrow a_{i, j-2}$. The associated cocycles are $\bar{\eta}_{1}: a_{2,0} \rightarrow 1, a_{1,1} \rightarrow 0, a_{0,2} \rightarrow 0$. Let $\alpha=\left[\bar{\eta}_{1}\right], \beta \in\left[\bar{\eta}_{2}\right]$ in $H^{2}(G, Z) . \bar{\eta}_{2}: a_{0,2} \rightarrow 1, a_{1,1} \rightarrow 0, a_{2,0} \rightarrow 0$. By inspection, we see that all 3-cocycles are multiples of $f: a_{03} \rightarrow 0, a_{12} \rightarrow-m / n, a_{21} \rightarrow 1, a_{30} \rightarrow 0$. Let $\xi=[f] \in H^{3}(G, Z)$. We see easily that $H^{*}(G, Z)=Z[\alpha, \beta, \xi)$ as ring. The only possible relations are of the form $\xi^{2}=p(\alpha, \beta)=$ polynomial in $\alpha$ and $\beta$. If $|G|$ is odd, then $\xi^{2}=0$. If $|G|$ is even, $\xi^{2}$ may or may not be zero.

EXAMPLE. $G=Z_{4} \oplus Z_{2}=\left(x, y: x^{4}=y^{2}=1, x y=y x\right)$.

Proposition 3.7. $H^{*}\left(Z_{4} \oplus Z_{2}, Z\right)=P[\alpha, \beta] \otimes E(\xi)$. That is, $4 \beta=2 \alpha=\xi^{2}=0$ are the only relations.

Proof. It is enough to show $\xi^{2}=0$. Let $j: Z \stackrel{\text { ept }}{\longrightarrow} Z_{2}$. This gives $j_{*}: H^{*}(G, Z)$ $\rightarrow H^{*}\left(G, Z_{2}\right)$, a ring morphism.

$$
H^{*}\left(Z_{4} \oplus Z_{2}, Z_{2}\right) \cong H^{*}\left(Z_{4}, Z_{3}\right) \otimes H^{*}\left(Z_{2}, Z_{2}\right)
$$

by Künneth. [17, p. 68] says that $H^{*}\left(Z_{2}, Z_{2}\right)=P\left[u_{1}\right]$, deg $u_{1}=1$. A simple-minded calculation, using [5, Chapter XII, §7], gives $H^{*}\left(Z_{4}, Z_{2}\right)=P\left[v_{2}\right] \otimes E\left(u_{2}\right)$, with 
$\operatorname{deg} u_{2}=1, \operatorname{deg} v_{2}=2$. In terms of our resolution, we may take representative cocycles

$u_{1}: a_{10} \rightarrow 1, \quad a_{01} \rightarrow 0, \quad u_{2}: a_{01} \rightarrow 1, \quad a_{10} \rightarrow 0, \quad v_{2}: a_{02} \rightarrow 1, \quad a_{n} \rightarrow 0, \quad a_{20} \rightarrow 0$.

Cupping with $u_{1}^{2}$ shifts a cocycle two units east. As $\xi$ is represented by $a_{12} \rightarrow-2$, $a_{21} \rightarrow 1, a_{03}, a_{30} \rightarrow 0$, clearly $j_{*}(\xi)=u_{2} u_{1}^{2}$. Therefore $j_{*}\left(\xi^{2}\right)=u_{2}^{2} u_{1}^{4}=0$. By inspection, $j_{*}: H^{6}(G, Z) \rightarrow H^{6}(G, Z)$ is mono except on powers of $\beta$. $\operatorname{Res}_{\langle x\rangle}(\xi)=0$, implies that $\xi^{2}$ is no such power. Hence $\xi^{2}=0$.

REMARK. In $\$ 4$ we will give a second proof of this fact, using the spectral sequence.

\section{Abelian, quaternion and dihedral groups.}

4.0. In [1, p. 61], Atiyah showed that if $Q=$ quaternions, then $H^{*}(Q, Z)$ $=Z[\alpha, \beta, \chi], \operatorname{deg} \alpha=\operatorname{deg} \beta=2, \operatorname{deg} \chi=4$, with relations $8 \chi=2 \alpha=2 \beta=\alpha^{2}=\beta^{2}=0$, $\alpha \beta=4 \chi$.

In [6], Evens stated that for $D=$ dihedral of order $8, H^{*}(D, Z)=Z[\alpha, \beta, \nu, \zeta]$, $\operatorname{deg} \alpha=\operatorname{deg} \beta=2, \operatorname{deg} \nu=3, \operatorname{deg} \zeta=4$, with relations $4 \zeta=2 \alpha=2 \beta=2 \nu=0, \nu^{2}=\alpha \zeta$, $\beta^{2}=\alpha \beta$.

4.1. R. G. Swan pointed out that using the Bockstein $\Delta$ it is easy to get $H^{*}\left(Z_{p} \oplus Z_{p}, Z\right)$. Indeed $H^{*}\left(Z_{p} \oplus Z_{p}, Z_{p}\right) \cong H^{*}\left(Z_{p}, Z_{p}\right) \otimes H^{*}\left(Z_{p}, Z_{p}\right)$ by Künneth. It is well-known that $H^{*}\left(Z_{p}, Z_{p}\right)=E(u) \otimes P(v), \operatorname{deg} u=1, \operatorname{deg} v=2, \Delta u=v$, ( $p$ odd), and $H^{*}\left(Z_{2}, Z_{2}\right)=P[v]$, deg $v=1, \Delta v=v^{2}$. The Bockstein arises from the sequence

$$
0 \longrightarrow Z \stackrel{p}{\longrightarrow} Z \stackrel{j}{\longrightarrow} Z_{p} \longrightarrow 0,
$$

which yields exact sequences

$$
0 \longrightarrow H^{k}\left(Z_{p} \oplus Z_{p}, Z\right) \longrightarrow H^{k}\left(Z_{p} \oplus Z_{p}, Z_{p}\right) \stackrel{\delta}{\longrightarrow} H^{k+1}\left(Z_{p} \oplus Z_{p}, Z\right) \longrightarrow 0
$$

(because $H^{*}\left(Z_{p} \oplus Z_{p}, Z\right)$ is of exponent $p$ in positive dimensions: Künneth gives us the additive structure of $\left.H^{*}\left(Z_{p} \oplus Z_{p}, Z\right)\right) . \Delta=j_{*} \circ \delta$. As $j_{*}$ is mono, $\operatorname{Ker} \Delta$ $=\operatorname{Ker} \delta=H^{*}\left(Z_{p} \oplus Z_{p}, Z\right) . \Delta$ is a derivation, and we can calculate $\operatorname{Ker} \Delta$ readily. As $j_{*}$ is a ring morphism we get:

Proposition 4.1. $H^{*}\left(Z_{p} \oplus Z_{p}, Z\right)=P[\alpha, \beta] \otimes E(\mu), \operatorname{deg} \alpha=\operatorname{deg} \beta=2, \operatorname{deg} \mu=3$, p odd $; H^{*}\left(Z_{2} \oplus Z_{2}, Z\right)=P[\alpha, \beta] \otimes Z[\mu], \operatorname{deg} \alpha=\operatorname{deg} \beta=2, \operatorname{deg} \mu=3$, with relations $\mu^{2}=\alpha \beta^{2}+\beta \alpha^{2}$.

In a similar fashion we find

Proposition 4.2. $H^{*}\left(Z_{p} \oplus Z_{p} \oplus Z_{p}, Z\right)=Z[\alpha, \beta, \gamma, \mu, \nu, \chi, \xi]$ ( $p$ odd), where $\operatorname{deg} \alpha=\operatorname{deg} \beta=\operatorname{deg} \gamma=2, \operatorname{deg} \mu=\operatorname{deg} \nu=\operatorname{deg} \chi=3, \operatorname{deg} \xi=4$. The relations are

$$
\begin{gathered}
\mu^{2}=\nu^{2}=\chi^{2}=\xi^{2}=0, \quad \alpha \nu+\beta \chi+\gamma \mu=0, \\
\gamma \mu=\alpha \xi, \quad \nu \chi=\gamma \xi, \quad \mu \nu=\beta \xi, \quad \mu \xi=\nu \xi=\chi \xi=0 .
\end{gathered}
$$


$H^{*}\left(Z_{2} \oplus Z_{2} \oplus Z_{2}, Z\right)=Z[\alpha, \beta, \gamma, \mu, \nu, \chi, \xi]$, where the degrees are the same and

$$
\begin{aligned}
& \mu^{2}=\alpha \beta^{2}+\beta \alpha^{2}, \quad \nu^{2}=\beta \gamma^{2}+\gamma \beta^{2}, \quad \chi^{2}=\gamma \alpha^{2}+\alpha \gamma^{2}, \\
& \alpha \nu+\beta \chi+\gamma \mu=0, \quad \xi^{2}=\alpha^{2} \beta \gamma+\beta^{2} \gamma \alpha+\gamma^{2} \alpha \beta, \\
& \chi \mu=\alpha \xi+\alpha \beta \gamma, \quad \nu \chi=\gamma \xi+\alpha \beta \gamma, \quad \mu \nu=\beta \xi+\alpha \beta \gamma, \\
& \mu \xi=\alpha \beta(\nu+\chi), \quad \nu \xi=\beta \gamma(\chi+\mu), \quad \chi \xi=\gamma \alpha(\mu+\nu) .
\end{aligned}
$$

4.2. Proposition 4.3. $H^{*}\left(Z_{p^{2}} \oplus Z_{p}, Z\right)=P[\alpha, \beta] \otimes E(\chi)$ for all $p$, where $\operatorname{deg} \alpha=\operatorname{deg} \beta=2, \operatorname{deg} \chi=3, p^{2} \alpha=p \beta=p \chi=0$.

Proof. Let $G=\left(x, y: x^{p^{2}}=y^{p}=1, x y=y x\right)$. Consider the spectral sequence of the extension $1 \rightarrow Z_{p^{2}} \rightarrow G \rightarrow Z_{p} \rightarrow 1 . E_{2}^{i, j} \cong H^{i}\left(Z_{p}, H^{j}\left(Z_{p^{2}}, Z\right)\right), E_{2}^{2,0} \cong Z_{p^{2}} \beta, E_{2}^{1,2} \cong Z_{p} \chi$. Using the multiplication rule in [3, Chapter XII, §7], we find that $\alpha, \beta, \chi$ generate $E_{2}$. Thus,

Also,

$$
E_{2}^{*, 0}=\sum_{i=0}^{\infty} Z \alpha^{i}, \quad E_{2}^{0, *}=\sum_{i=0}^{\infty} Z \beta^{i}, \quad E_{2}^{*, 2}=\sum_{i=0}^{\infty} Z \alpha^{i} \beta \oplus Z \alpha^{i} \chi .
$$

$$
\alpha: E_{2}^{i, j} \stackrel{\cong}{\longrightarrow} E_{2}^{2+2, j} \quad(i, j \geqq 0), \quad \beta: E_{2}^{i, j} \cong E_{2}^{i, j+2} \quad(i \geqq 0, j>0) .
$$

As the extension is split, the terms on the base are never hit. (See $\$ 1.3$, Corollary I.) This means that $\alpha, \beta, \chi$ are universal cycles, whence $E_{2}=E_{\infty}$. If $p$ odd, then $\operatorname{deg} \chi=3$ implies that $\chi^{2}=0$ in $H^{*}(G, Z)$. Thus for $p$ odd we have our result. If $p=2$, we must prove $\chi^{2}=0$. In both cases we will be very explicit about $\alpha$ and $\beta$. We choose $\alpha=\inf \chi_{y} \in H^{2}\left(Z_{2}, Z\right), \beta=\inf \chi_{x} \in H^{2}\left(Z_{4}, Z\right), \chi_{x}, \chi_{y}$ being maximal generators. As $H^{2}(G, Z) \cong{ }^{\delta} \operatorname{Hom}(G, Q / Z)$, and as Inf and $\delta$ commute, we can take $\alpha: x \rightarrow 0, y \rightarrow 1 / p, \beta: x \rightarrow 1 / p^{2}, y \rightarrow 0$. Now let $p=2$. Define $\lambda \in$ Aut $\left(Z_{4} \oplus Z_{2}\right)$ by $\lambda: y \rightarrow y x^{2}, x \rightarrow x y$. We find that $\alpha^{\lambda}=\alpha+2 \beta, \beta^{\lambda}=\beta+\alpha$, where we let $\lambda$ also designate the induced ring automorphism of $H^{*}\left(Z_{4} \oplus Z_{2}, Z\right)$. As $H^{3}(G, Z)=Z_{2} \chi$, we must have $\chi^{\lambda}=\chi$. Since $\chi^{2}=0$ in $E_{2}=E_{\infty}$, any relation will be of the form $\chi^{2}$ $=a \alpha \beta^{2}+b \alpha^{2} \beta+c \alpha^{3}$. Apply $\lambda$ to this. We find

$$
\begin{aligned}
\chi^{2}=a \alpha \beta^{2}+b \alpha^{2} \beta+c \alpha^{3} & =a(\alpha+2 \beta)\left(\beta^{2}+\alpha^{2}\right)+b(\alpha+2 \beta)^{2}(\beta+\alpha)+c(\alpha+2 \beta)^{3} \\
& =2 a \beta^{3}+a \alpha \beta^{2}+b \alpha^{2} \beta+(a+b+c) \alpha^{3} .
\end{aligned}
$$

As $\beta^{3}, \alpha^{2} \beta, \alpha \beta^{2}, \alpha^{3}$ are linearly independent in $E_{2}=E_{\infty}$, hence $\left({ }^{2}\right)$ in $H^{*}(G, Z)$, we conclude that $a+b \equiv 0$ (2), $2 a \equiv 0$ (4). Hence $a \equiv b \equiv 0$ (2). So our relation boils down to $\chi^{2}=c \alpha^{3}$. But a glance at the spectral sequence of $0 \rightarrow Z_{2} \rightarrow G \rightarrow Z_{4} \rightarrow 0$ shows that $\operatorname{Res}_{z_{2}}(\chi)=0, \operatorname{Res}_{z_{2}}(\alpha)=\chi_{y}$. Applying $\operatorname{Res}_{z_{2}}$ to our relation thus gives: $0=c \chi_{y}^{3}$, implying $c=0$. Thus $\chi^{2}=0$ as desired. Q.E.D.

REMARK 4.4. In the above discussion we have used $\alpha, \beta, \chi$ to denote elements of $E_{2}=E_{\infty}$ and elements of $H^{*}(G, Z)$ which map onto the former under the projection maps $\pi_{r}$ in the exact sequences

$$
0 \rightarrow H^{i}(G, Z)_{r+1} \rightarrow H^{i}(G, Z)_{r} \rightarrow E_{\infty}^{r, i-r} \rightarrow 0 .
$$

We will continue this convenient abuse of notation in future sections.

(2) As $\beta^{i}=\inf \chi_{x}^{i}$, we know that $p^{2} \beta^{i}=0$ in $H^{*}(G, Z)$. 
5. Groups of type I. C. T. C. Wall has calculated the additive structure of $H^{*}(G, Z)$, where $G$ is a split extension of cyclic groups [16], as follows:

Proposition 5.1 (WALl). Let $G=\left(x, y: x^{r}=y^{s}=1, y^{-1} x y=x^{t}\right)$. Then $H^{2 i}(G, Z)$ $=Z_{s} \oplus \sum_{k<i} Z_{q_{k}} \oplus Z_{h_{i}}, \quad$ where $h_{i}=\left(t^{i}-1, r\right), \quad k_{i}=\left(\sum_{j=0}^{s-1} t^{i j}, r\right), \quad H^{2 i+1}(G, Z)$ $=\sum_{k<i} Z_{q_{k}}$ and $q_{i}=h_{i} k_{i} / r$.

Wall also noted that in the spectral sequence of

$$
1 \rightarrow Z_{r} \rightarrow G \rightarrow Z_{s} \rightarrow 1,
$$

$E_{2}=E_{\infty}$. I will consider the special case $r=p^{2}, s=p, t=1+p$. As $p=2$ implies $G=D$, I will suppose $p$ odd. As

$k_{i}=p$, so

$$
\begin{array}{rl}
h_{i}=p & \text { if } p \nmid i, \\
=p^{2} & \text { if } p \mid i, \\
q_{i}=1 & p \nmid i, \\
=p & p \mid i .
\end{array}
$$

Proposition 5.1 now gives us the additive structure. In particular, $H^{2 i}(G, Z)$ $=Z_{p} \oplus Z_{p}$ if $i<p,=Z_{p} \oplus Z_{p^{2}}$ if $i=p$, and $H^{2 i+1}(G, Z)=0$ for $0<i<p$. Let $H=\langle x\rangle, K=\langle y\rangle$. We have $E_{2}^{i, j} \cong H^{i}\left(K, H^{j}(H, Z)\right) . H^{*}(H, Z)=P[\beta], \operatorname{deg} \beta=2$, $p^{2} \beta=0$. As $H \triangleleft G, y$ acts on $H^{*}(H, Z) . H^{2}(H, Z) \cong{ }^{\delta} \operatorname{Hom}(H, Q / Z)$, and the action of $y$ commutes with $\delta . y^{-1} x y=x^{1+p}$.

Let $\beta: H \rightarrow Q / Z$. $\beta(x)=1 / p^{2}$. Then

$$
y \beta(x)=y \cdot \beta\left(y^{-1} x y\right)=(1+p) / p^{2} .
$$

Thus $y \beta=(1+p) \beta$, and $y \beta^{i}=(1+p)^{i} \beta^{i}=(1+p i) \beta^{i}$, since $p^{2} \beta=0$. Thus, we find:

where $\beta_{i}=p \beta^{i}$.

$$
\begin{aligned}
H^{i}(H, Z)^{K} & =\beta^{i} & & \text { if } p \mid i, \\
& =\beta_{i} & & \text { if } p \nmid i,
\end{aligned}
$$

By periodicity,

$$
\begin{aligned}
H^{i}\left(K, H^{j}(H, Z)\right) & \stackrel{\phi}{\cong} \hat{H}^{0}\left(K, H^{j}(H, Z)\right) \quad i \text { even, } \\
& \stackrel{\phi}{\cong} \hat{H}^{1}\left(K, H^{j}(H, Z)\right) \quad i \text { odd, }
\end{aligned}
$$

where $\phi=\alpha \cup, \alpha \in H^{2}(K, Z)$, a maximal generator. As $N_{y} H^{j}(H, Z)=N_{y}\left(\beta^{i}\right)=p \beta^{i}$, so $\operatorname{Ker} N_{y}=p \beta^{i}$. Hence

where $p \bar{\beta}=0$.

$$
\begin{array}{rlrl}
\hat{H}^{0}\left(K, H^{j}(H, Z)\right) & =0, & & \text { if } p \nmid i, \\
& =Z \tilde{\beta}^{i}, & \text { if } p \mid i,
\end{array}
$$

$$
\begin{aligned}
\hat{H}^{1}\left(K, H^{j}(H, Z)\right) & =0, & & \text { if } p \nmid i, \\
& =p Z \beta^{i}, & & \text { if } p \mid i .
\end{aligned}
$$

Applying $\phi$, we get: (setting $\zeta=\beta^{p}$ ) (and letting $\chi \in E_{2}^{1,2 p}$ be a generator) $E_{2}^{0,2 j}$ $=Z \beta_{j_{0}} \zeta^{a}$, where $j=j_{0}+a p, 0 \leqq j_{0}<p, E_{2}^{i, j}=0$ if $2 p \nmid j$ or $j$ odd, $i \geqq 0 . E_{2}^{*, 0}=\sum_{i=0}^{\infty} Z \alpha^{i}$, $E_{2}^{i .2 p j}=Z \alpha^{i / 2} \zeta^{j-1}, i$ even, $=Z \chi \alpha^{(i-1) / 2} \zeta^{j-1}, i$ odd, $j>0$. 
We can now see that $E_{2}=E_{\infty}$. For as extension $\left({ }^{*}\right)$ is split, the terms on the base are never hit. Thus, $\alpha, \beta_{1}, \ldots, \beta_{p-1}, \zeta, \chi$ are all universal cycles, and generate $E_{2}$. Therefore $E_{2}=E_{\infty} \cdot \chi$ is of odd degree, so $\chi^{2}=0$. In $E_{2}=E_{\infty}, \beta_{2} \chi=\beta_{i} \beta_{j}=0$, all $i, j$, and $p^{2} \zeta=0$. I claim $p^{2} \zeta=0$ in $H^{*}(G, Z)$. For if not then $\zeta$ would be a maximal generator, implying $G$ is periodic [3, Chapter XII, §11]. Contradiction. Done.

Conceivably, $\beta_{i} \beta_{j}=d_{i, j} \alpha^{i+j}, d_{i, j} \neq 0$, or $\beta_{i} \chi=d_{i} \alpha^{i} \chi, d_{i} \neq 0$. To investigate, we use the restriction-corestriction sequences. From our $E_{\infty}$ term we find

$$
\begin{aligned}
\left|H^{i}(G, Z)\right| & =0 \quad i \text { odd, } \quad 0<i<2 p . \\
& =p^{2} \quad i \text { even, }
\end{aligned}
$$

Proposition 2.1 yields

$$
\begin{aligned}
0 \longrightarrow H^{2 i-1}(G, Z) & \longrightarrow H^{2 i}(G, X) \longrightarrow H^{2 i}(H, Z) \\
& \stackrel{\text { Cor }}{\longrightarrow} H^{2 i}(G, Z) \longrightarrow H^{2 i+1}(G, X) \longrightarrow 0
\end{aligned}
$$

and

$$
\begin{aligned}
0 \longrightarrow H^{2 i-1}(G, X) & \longrightarrow H^{2 i}(G, \dot{Z}) \stackrel{\text { Res }}{\longrightarrow} H^{2 i}(H, Z) \\
& \longrightarrow H^{2 i}(G, X) \longrightarrow H^{2 i+1}(G, Z) \longrightarrow 0 .
\end{aligned}
$$

As $\operatorname{Im}\left(\operatorname{Res}_{2 i}\right)=Z \beta_{i}$, of order $p$ (cf. Corollary II, §1), we get

$$
\left|H^{2 i-1}(G, X)\right|=p, \quad\left|H^{2 i}(G, X)\right|=p, \quad 1 \leqq i<p .
$$

Hence $\left|\operatorname{Im}\left(\operatorname{Cor}_{2 i}\right)\right|=p \quad(1 \leqq i<p)$. Thus $\operatorname{Cor} \beta^{i} \neq 0, p \operatorname{Cor} \beta^{i}=0 \quad(1 \leqq i<p)$. The formula $\operatorname{Cor}(\operatorname{Res}(a) \cdot b)=a \operatorname{Cor} b$ [3, Chapter XII, §8], and Res $\alpha=0$, implies $\alpha \operatorname{Cor} \beta^{i}=0$. And Res $\operatorname{Cor} \beta^{i}=N_{y} \beta^{i}=p \beta^{i}=\beta_{i}$. Thus, in $H^{*}(G, Z)$ we can choose $\beta_{i}=\operatorname{Cor} \beta^{i}$ (see Remark 4.4), so that $p \beta_{i}=0, \alpha \beta_{i}=0$ in $H^{*}$. Now suppose $\beta_{i} \beta_{j}=d \alpha^{i+j}$. Thus implying $0=\alpha \beta_{i} \beta_{j}=d \alpha^{i+j+1}$ which implies $d=0$. And if $\beta_{i} \chi=d \alpha^{i} \chi$, then $0=\alpha \beta_{i} \chi=d \alpha^{i+1} \chi$. As $\alpha^{i+1} \chi \neq 0$ in $H^{*}$, so $d=0$. We summarize our results in the following

THEOREM 5.2. If $G=\left(x, y: x^{p^{2}}=y^{p}=1, y^{-1} x y=x^{1+p}\right)$, then the ring $H^{*}(G, Z)$ $=Z\left[\alpha, \chi, \zeta, \beta_{1}, \ldots, \beta_{p-1}\right], \operatorname{deg} \alpha=2, \operatorname{deg} \beta_{i}=2 i, \operatorname{deg} \zeta=2 p, \operatorname{deg} \chi=2 p+1$, with relations $p^{2} \zeta=p \alpha=p \chi=p \beta_{i}=0, \chi^{2}=0, \beta_{i} \alpha=\beta_{i} \chi=\beta_{i} \beta_{j}=0$, all $i, j$. If $\beta \in H^{2}(\langle x\rangle, Z)$ is a maximal generator, we may take $\beta_{i}=\operatorname{Cor} \beta^{i}$.

\section{Groups of type II.}

6.1. Let $G=\left(A, B: A^{p}=B^{p}=[A, B]^{p}=[A,[A, B]]=[B,[A, B]]=1\right)$. Let $C=$ $[A, B]=B^{-1} A^{-1} B A$. Thus $A^{-1} B A=B C$. Set $\alpha_{j}=\left|\hat{H}^{j}(G, Z)\right|$. Our calculation of $H^{*}(G, Z)$ will proceed in leisurely steps.

Step 1. A proof of the inequality $\alpha_{j+2 p} \leqq p^{p+1} \alpha_{j}$, all $j$. Also, the observation that for $i \leqq p, \alpha^{i}, \alpha^{i-1} \beta, \ldots, \beta^{i}$ are linearly independent, and that for $i<p, \alpha^{i} \mu, \alpha^{i-1} \beta \mu, \ldots$, $\alpha \beta^{i-1} \mu$ are likewise linearly independent. (Here, $\alpha, \beta \in H^{2}(G, Z), \mu \in H^{3}(G, Z)$ are certain cohomology classes.) 
Step 2. The calculation of the $E_{2}$ terms of the spectral sequences of the extensions

$$
1 \rightarrow\langle c\rangle \rightarrow G \rightarrow\langle\bar{A}, \bar{B}\rangle \rightarrow 1
$$

and

$$
1 \rightarrow H \rightarrow G \rightarrow\langle A\rangle \rightarrow 1 \quad \text { (split), }
$$

where $H=\langle B, C\rangle$.

Step 3. Using the restriction-corestriction sequences, we get enough information to calculate the $E_{\infty}$ terms.

Step 4. By observing the effect of automorphisms on the generators of $H^{*}$, we find the relations.

6.2. Let $H=\langle B, C\rangle, H \triangleleft G$. This has a 1-dimensional unitary representation as follows: $B$ acts as identity, $C$ as multiplication by $\zeta$, where $\zeta=e^{2 \pi i / p}$. Form $C G \otimes_{H} C$, the induced representation. $1 \otimes 1, A \otimes 1, \ldots, A^{p-1} \otimes 1$ form a basis. For this basis, $A, B, C$ have matrices

$$
\begin{aligned}
& A \sim\left(\begin{array}{lllll}
0 & 1 & 0 & \cdots & 0 \\
0 & 0 & 1 & \cdots & 0 \\
0 & 0 & \cdots & \cdots & 1 \\
1 & 0 & \cdots & \cdots & 0
\end{array}\right), \quad B \sim\left(\begin{array}{llllll}
1 & & & & & \\
& \zeta & & & & \\
& & \zeta^{2} & & & \\
& & & . & \\
& & & & \cdot \\
& & & & \zeta^{p-1}
\end{array}\right), \\
& C \sim\left(\begin{array}{llll}
\zeta & & & \\
& \zeta & & \\
& & \cdot & \\
& & & \cdot \\
& & & \zeta
\end{array}\right)
\end{aligned}
$$

The induced representation is again unitary. Thus $H$ acts on $S^{1} \subset C$, and $G$ acts on $S^{2 p-1} \subset C^{p}$, with $C$ acting freely. Using the fact $S^{2 p-1}=S^{1} * \cdots * S^{1}$ (p-fold join), we will construct a $G$-invariant decomposition for $S^{2 p-1}$. In $S^{1}$, take $e=1$, $f=\{z \mid 0 \leqq \arg z \leqq 2 \pi / p\}$ as generating cells for an $H$-invariant decomposition. Thus the 0 -cells are $e, \zeta e, \ldots, \zeta^{p-1} e$, the 1 -cells $f, \zeta f, \ldots, \zeta^{p-1} f$, and set $\partial f=\zeta e-e$. Let $C\left(S^{1}\right)$ be the corresponding complex, i.e. $C\left(S^{1}\right)=\left\{C_{0} \longleftarrow C_{1}\right\}$. If $C\left(S^{1}\right)=$ augmented complex with dimensions raised by 1 , form $\dot{C}\left(S^{2 p-1}\right)=\dot{C}\left(S^{1}\right) \otimes \cdots \otimes \dot{C}\left(S^{1}\right)$ $=p$-fold tensor product. Finally, set $C\left(S^{2 p-1}\right)=$ deaugmented complex of $\dot{C}^{\prime}\left(S^{2 p-1}\right)$, with dimensions lowered by 1 . As in $\S 3, C\left(S^{2 p-1}\right)$ is a $G$-complex and is $C$-projective (actually $C$-free). Take $G=\bigcup_{i=0}^{p-1} A^{i} H$, yielding the monomorphism $\Phi: G \rightarrow \Sigma_{p} \prec H$. $C\left(S^{2 p-1}\right)$ is made a $G$-complex via $\Phi$. If $x_{1} \otimes \cdots \otimes x_{p}$ is a typical decomposable element, then $C\left(x_{1} \otimes \cdots \otimes x_{p}\right)=\zeta x_{1} \otimes \cdots \otimes \zeta x_{p}, B\left(x_{1} \otimes \cdots \otimes x_{p}\right)=x_{1} \otimes$ $\zeta x_{2} \otimes \cdots \otimes \zeta^{p-1} x_{p}, \quad A\left(x_{1} \otimes \cdots \otimes x_{p}\right)=(-1)^{v} x_{p} \otimes x_{1} \otimes \cdots \otimes x_{p-1} \quad$ where $\quad \nu$ $=\operatorname{deg} x_{p}\left(\sum_{i=1}^{p-1} \operatorname{deg} x_{i}\right)$. Write

$$
C\left(S^{2 p-1}\right)=\left\{C_{0} \leftarrow C_{1} \leftarrow \cdots \leftarrow C_{p-1} \leftarrow \cdots \leftarrow C_{2 p-1}\right\} .
$$


We will examine the modules $C_{i}$, and will show that they are $G$-free except for $C_{0}, C_{1}, C_{p-1}$ and $C_{2 p-1}$. Let $x_{1} \otimes \cdots \otimes x_{p} \in C_{n}$ be a decomposable element. Thus, each $x_{i}$ is 1 , or $e$, or $f$. The elements

$$
B^{i} C^{j} x_{1} \otimes \cdots \otimes x_{p}=\zeta^{j} x_{i} \otimes \zeta^{i+j} x_{2} \otimes \zeta^{2 i+j} x_{3} \otimes \cdots \otimes \zeta^{(p-1) i+j} x_{p}
$$

will be distinct $(1 \leqq i, j<p)$, hence $Z$-independent, unless $\zeta^{k i+j} x_{k+1}=x_{k+1}(0 \leqq k<p)$. As the $\{k i+j\}$ are incongruent $\bmod p$, precisely one is divisible by $p$, say $k_{0} i+j \equiv 0$ $(p)$. Thus $x_{r}=1$ for $r \neq k_{0}$, hence

$$
x_{1} \otimes \cdots \otimes x_{p}=1 \otimes 1 \otimes \cdots \otimes x_{k_{0}} \otimes \cdots \otimes 1,
$$

with $x_{k_{0}}=e$ or $f$. Thus our element lies in $C_{0}$ or in $C_{1}$. It is easy to find the structure of these modules. If $\Lambda=Z G$, then $C_{0}=\Lambda(e \otimes 1 \otimes \cdots \otimes 1)$. As $B(e \otimes \cdots \otimes 1)$ $=e \otimes \cdots \otimes 1$, we see that $C_{0} \cong Z(G /\langle B\rangle)$. (Here, if $\pi \subset$ II, a subgroup, then $Z(\mathrm{II} / \pi)=\{x \pi \mid x \in \mathrm{II}\}=$ left coset space, as a left II-module.) $C_{1}$ has elements of two sorts: $\zeta^{i} e \otimes 1 \otimes \cdots \otimes 1 \otimes \zeta^{j} e \otimes 1 \otimes \cdots \otimes 1$, and $f \otimes 1 \otimes \cdots \otimes 1$. An element of the first type generates a $G$-module $\cong \Lambda$, and as before, $\Lambda(f \otimes 1 \otimes \cdots \otimes 1)$ $\cong Z G /\langle B\rangle$. Thus $C_{1} \cong Z G /\langle B\rangle \oplus F$, with $F G$-free.

Now suppose the $B^{i} C^{j} x_{1} \otimes \cdots \otimes x_{p}$ are all distinct. If the $A^{k} B^{i} C^{j} x_{1} \otimes \cdots \otimes x_{p}$ are all distinct, then $\Lambda x_{1} \otimes \cdots \otimes x_{p} \cong \Lambda$. The only $x_{1} \otimes \cdots \otimes x_{p}$ for which this might fail must have all $x_{i}=e$ or all $x_{i}=f$, i.e. must lie in $C_{p-1}$ or $C_{2 p-1}$ (whence, for $i \neq 0,1, p-1,2 p-1, C_{i}$ is $G$-free). Consider $x=\zeta^{a_{1}} e \otimes \zeta^{a_{2}} e \otimes \cdots \otimes \zeta^{a_{p}} e \in C_{p-1}$. Note that on such elements $A$ acts with sign +1 (this is essential to our calculation, and depends on the fact that $p$ is odd). Thus if the $A^{k} B^{i} C^{j} x$ are not all distinct, then $A^{k} B^{i} C^{j} x=x$, some $k, i, j$. Thus, $\left(A^{k} B^{i} C^{j}\right)^{r} x=x$. (Note $k \neq 0$.) $\left(A^{k} B^{i} C^{j}\right)^{r}=$ $A^{k r} B^{i r} C^{r(r-1) k i / 2+j r}$. Choose $r \ni k r \equiv 1(p)$. In other words, we may suppose $k=1$. $A B^{i} C^{j} x=x$ implies $B A B^{i-1} C^{j} B x=B x$ or $A B^{i} C^{j+1} B x=B x$. So replacing $x$ by $B^{-j} x$ let us assume $j=0$, or $A B^{i} x=x$. $x$ can be multiplied by a power of $C$, and the relation still holds. So we can suppose $a_{1}=0$. Writing our equation as $B^{i} x=A^{p-1} x$, we see that

$$
e \otimes \zeta^{a_{2}+i} e \otimes \zeta^{a_{3}+2 i} e \otimes \cdots \otimes \zeta^{a_{p}+(p-1) i} e=\zeta^{a_{2}} e \otimes \zeta^{a_{3}} e \otimes \cdots \otimes \zeta^{a_{p}} e \otimes e
$$

implies

or

$$
a_{2}=0, a_{3}=i, a_{4}=3 i, \ldots, a_{p}=C_{p-1,2} i,
$$$$
x=e \otimes e \otimes \zeta^{i} e \otimes \zeta^{3 i} e \otimes \cdots \otimes \zeta^{C_{p-1,2}} e .
$$

For $i=0,1, \ldots, p-1$, we get just $p$ nonfree components of $C_{p-1}$, so

$$
C_{p-1} \cong Z G \mid\langle A\rangle \oplus Z G /\langle A B\rangle \oplus \cdots \oplus Z G /\left\langle A B^{p-1}\right\rangle \oplus F, \quad F G \text {-free. }
$$

The case of $C_{2 p-1}$ is entirely similar. At last we can compute. Consider

$$
0 \leftarrow Z \leftarrow C_{0} \leftarrow C_{1} \leftarrow \cdots \leftarrow C_{2 p-1} \leftarrow Z \leftarrow 0,
$$

and let $X, Y, V, W$, and $U$ be the image-kernels at $C_{0}, C_{1}, C_{p-2}, C_{p-1}$, and $C_{2 p-1}$, 
respectively. Applying Tate cohomology to the resulting exact sequences, and using $\hat{H}^{i}(\mathrm{II}, Z(\mathrm{II} / \pi)) \approx \hat{H}^{i}(\pi, Z)$, [3, p. 196], we get, for $i$ odd, four long exact sequences, plus two-dimensional shifts, for every $i$.

( $i$ odd):
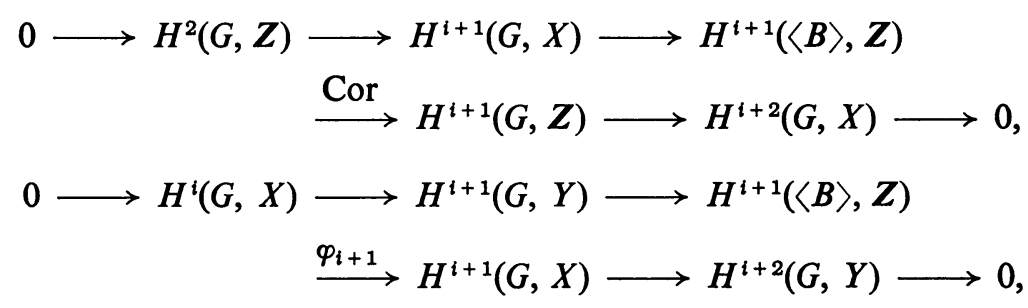

$0 \longrightarrow H^{i}(G, V) \longrightarrow H^{i+1}(G, W) \stackrel{\psi_{i+1}}{\longrightarrow} \sum_{t=0}^{p-1} H^{i+1}\left(\left\langle A B^{t}\right\rangle, Z\right)$

$$
\longrightarrow H^{i+1}(G, V) \longrightarrow H^{i+2}(G, W) \longrightarrow 0,
$$

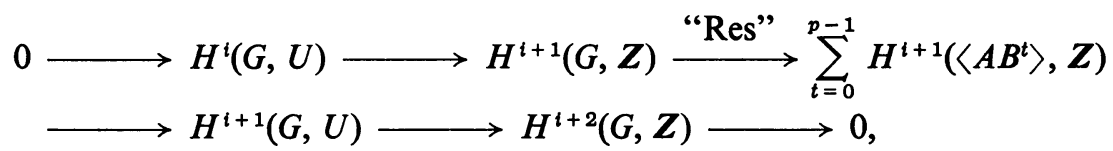

$H^{i}(G, Y) \cong H^{i+p-3}(G, V), \quad H^{i}(G, W) \cong H^{i+p-1}(G, U), \quad$ all $i$.

Here

$$
\text { “'Res" maps } \xi \rightarrow\left(\operatorname{Res}_{L_{0}}(\xi), \operatorname{Res}_{L_{1}}(\xi), \ldots, \operatorname{Res}_{L_{p-1}}(\xi)\right),
$$

where $L_{t}=\left\langle A B^{t}\right\rangle$. Observe that for $i$ odd, $H^{i+1}(\langle B\rangle, Z) \cong H^{i+1}\left(L_{t}, Z\right) \cong Z_{p}$. So with $\alpha_{j}=\left|H^{t}(G, Z)\right|$ we get

$$
\begin{aligned}
\left|H^{i+2}(G, Z)\right| & \leqq\left|H^{i+1}(G, U)\right|=\left|H^{i-p+2}(G, W)\right| \leqq p^{p}\left|H^{i-p+1}(G, V)\right| \\
& =p^{p}\left|H^{i-2 p+4}(G, Y)\right| \leqq p^{p}\left|H^{i-2 p+3}(G, X)\right| \\
& \leqq p^{p+1}\left|H^{i-2 p+2}(G, Z)\right|, \quad(i \text { odd }), \text { or } \alpha_{i+2} \leqq p^{p+1} \alpha_{i-2 p+2} .
\end{aligned}
$$

In exactly the same way we show that this holds for $i$ even. Hence

$$
\alpha_{j+2 p} \leqq p^{p+1} \alpha_{j} \text { for all } j
$$

For $j>0$, this is an equality. However, I have not been able to prove this directly. It is easy to see the following:

(1) Cor $=0$ in positive dimensions,

(2) $\varphi_{i+1}=0$ for $i$ odd, $i>0$,

(3) "Res" is onto for $i \geqq 2 p-3, i$ odd.

The trouble is that I have not been able to show that $\psi_{i+1}$ is onto in a suitable range. As I have no further use for the above sequences, I will limit myself to a discussion of the map "Res", and will estimate |Im ("Res")|. 
Proposition 6.1. If $\alpha, \beta \in H^{2}(G, Z)$ are the inflations of maximal generators $\chi_{A} \in H^{2}(\langle A\rangle, Z), \chi_{B} \in H^{2}(\langle B\rangle, Z)$, respectively, then $\alpha^{n}, \alpha^{n-1} \beta, \ldots, \beta^{n} \in H^{2 n}(G, Z)$ are linearly independent, for $1 \leqq n \leqq p$.

Proof. Let $L_{t}=\left\langle A B^{t}\right\rangle,(0 \leqq t<p)$. Set $L_{p}=\langle B\rangle$. As $H^{2}(G, Z) \cong{ }^{\delta} \operatorname{Hom}(G, Q / Z)$, we will take $\alpha, \beta$ corresponding to $\alpha: A \rightarrow 1 / p, B \rightarrow 0, \beta: A \rightarrow 0, B \rightarrow 1 / p$. Let $\chi_{t} \in H^{2}\left(L_{t}, Z\right) \cong_{\delta} \operatorname{Hom}\left(L_{t}, \boldsymbol{Q} / Z\right)$ correspond to $A B^{t} \rightarrow 1 / p$ if $0 \leqq t<p$, and to $B \rightarrow 1 / p$ if $t=p \cdot \chi_{t}$ is a maximal generator. Let $a_{t}=\alpha-t^{-1} \beta, t \neq 0, a_{0}=\beta, a_{p}=\alpha$.

$$
\begin{array}{rlrl}
\operatorname{Res}_{L_{j}} a_{p}=\operatorname{Res}_{L_{j}}(\alpha) & =\chi_{j} & j \neq p, \\
& =0 & j=p, \\
\operatorname{Res}_{L_{j}}\left(a_{0}\right)=\operatorname{Res}_{L_{j}}(\beta) & =j \chi_{j} & j \neq p, \\
& =\chi_{p} & j=p .
\end{array}
$$

Hence

$$
\begin{array}{rlrl}
\operatorname{Res}_{L_{j}}\left(a_{t}\right) & =\left(1-j t^{-1}\right) \chi_{j} & j \neq p, t \neq 0, p, \\
& =-t^{-1} \chi_{p} & j=p, t \neq 0, p .
\end{array}
$$

Thus we see that $\operatorname{Res}_{L_{j}}\left(a_{t}\right)=0$ if and only if $j=t$, for all $j, t$. Recall that the powers of a maximal generator are again maximal generators. Let $I \subset\{0,1, \ldots, p\}$ be a subset $\ni|I|=n, 1 \leqq n \leqq p$. Form $a_{I}=\prod_{i \in I} a_{i}$. We have: $\operatorname{Res}_{L,}\left(a_{I}\right)=0$ if and only if $j \in I$. Choosing $I_{j}=\{0, \ldots, \hat{j}, \ldots, n\}$ clearly $\left\{a_{I_{0}}, \ldots, a_{I_{n}}\right\}$ is a set of $n+1$ linearly independent elements of $H^{2 n}(G, Z)$. As it is a subset of the span of $\left\{\alpha^{n}, \alpha^{n-1} \beta, \ldots, \beta^{n}\right\}$, the latter set must consist of linearly independent elements. Q.E.D.

For smoothness of exposition, we will invoke the following theorem:

THEOREM 6.2 (KuO). If $G$ is a group of odd order, $l \geqq 0, e=$ exponent of $H^{2 l+1}(G, Z)$, then $e^{2}$ divides the order of $G$.

Proof. Tze-Nan Kuo, Thesis, University of Chicago, 1966.

Applying this result to our group of order $p^{3}$, we get $p H^{2 l+1}(G, Z)=0, l \geqq 0$.

Proposition 6.3. $H^{3}(G, Z) \cong Z_{p} \oplus Z_{p}, H^{4}(G, Z) \cong Z_{p} \oplus Z_{p} \oplus Z_{p} \oplus Z_{p}$.

Proof. By Proposition 6.1, $H^{2}(G, Z) \stackrel{\alpha}{\rightarrow} H^{4}(G, Z)$ is mono. Using the second form of the Res-Cor sequences, we get

$$
0=H^{1}(G, Z) \stackrel{\alpha}{\longrightarrow} H^{3}(G, Z) \stackrel{\rho}{\longrightarrow} T^{3} \stackrel{\tau}{\longrightarrow} H^{2}(G, Z) \stackrel{\alpha}{\longrightarrow} H^{4}(G, Z)
$$

implies $H^{3}(G, Z) \approx T^{3}$. Also (with $K=\langle A\rangle$ ),

$$
0 \rightarrow H^{2}(H, Z)_{K} \rightarrow T^{3} \rightarrow H^{3}(H, Z)^{K} \rightarrow 0 .
$$

As $H^{*}(H, Z)=P[\beta, \gamma] \otimes E(\mu)$, where $\beta: B \rightarrow 1 / p, C \rightarrow 0, \gamma: B \rightarrow 0, C \rightarrow 1 / p$, with $k \beta=\beta, k \gamma=\gamma+\beta, k \mu=\mu, k$ being the automorphism of $H^{*}(H, Z)$ induced by $A$, we find that $H^{2}(H, Z)_{K}=Z \gamma, H^{3}(H, Z)^{K}=Z \mu$, both of order $p$, implies $p^{2}=\left|T^{3}\right|$ $=\left|H^{3}(G, Z)\right|$, and Res: $H^{3}(G, Z) \rightarrow H^{3}(H, Z)$ is epi. Choose $\mu \in H^{3}(G, Z)$, 
Res $\mu=\mu$ (convenient abuse of notation), $\nu \in H^{3}(G, Z)$, Res $\nu=0$. Clearly $p \nu=0$. Miss Kuo's Theorem implies that $p \mu=0$. Hence $H^{3}(G, Z) \approx Z_{p} \oplus Z_{p}$, with basis $\{\mu, \nu\}$. As $p \mu=\operatorname{Cor} \operatorname{Res} \mu=\operatorname{Cor} \mu$, we see that $\operatorname{Cor}: H^{3}(H, Z) \rightarrow H^{3}(G, Z)$ is zero.

Claim $\left|H^{4}(G, Z)\right|=p^{4}$. For using the first form of Res-Cor sequences:

$$
H^{3}(G, Z) \stackrel{\text { Res }}{\longrightarrow} H^{3}(H, Z) \longrightarrow H^{3}(X) \longrightarrow H^{4}(G, Z) \stackrel{\text { Res }}{\longrightarrow} H^{4}(H, Z) .
$$

The first Res is epi, the second has image $Z \beta^{2}$, of order $p$. Hence $\left|H^{4}(G, Z)\right|$ $=p\left|H^{3}(G, X)\right|$. But

$$
H^{2}(H, Z) \stackrel{\text { Cor }}{\longrightarrow} H^{2}(G, Z) \longrightarrow H^{3}(G, X) \longrightarrow H^{3}(H, Z) \stackrel{\text { Cor }}{\longrightarrow} H^{3}(G, Z),
$$

exact. By the formula for Cor in $\S 1$, we see that $\operatorname{Cor}=0$ in dimension 2 .

We noted above that $\mathrm{Cor}=0$ in dimension 3. As $\left|H^{2}(G, Z)\right|=p^{2},\left|H^{3}(H, Z)\right|=p$, so $\left|H^{3}(G, X)\right|=p^{3}$, and $\left|H^{4}(G, Z)\right|=p^{4}$. By Proposition 6.1, $\alpha^{2}, \alpha \beta, \beta^{2}$ are independent. Let $\chi \in H^{4}(G, Z)$ be a further generator.

Claim $p \chi=0$. For if $p \chi=a \alpha^{2}+b \alpha \beta+c \beta^{2}$, then $p \chi \alpha=0$. This implies that $a \alpha^{3}+b \alpha^{2} \beta$ $+c \alpha \beta^{2}=0$. As $p \geqq 3$, Proposition 6.1 implies that $a=b=c=0$. So $p \chi=0$ and hence $H^{4}(G, Z) \approx Z_{p} \oplus Z_{p} \oplus Z_{p} \oplus Z_{p}$, with basis $\left\{\alpha^{2}, \alpha \beta, \beta^{2}, \chi\right\}$. Q.E.D.

Remark 1. The author checked Proposition 6.3 by calculating with an explicit resolution. It was a horrible experience.

Remark 2. Theorem 6.2 was needed only to show that $p H^{3}(G, Z)=0$. We will prove this by a different method below, so that Theorem 6.2 is not really necessary in proving Proposition 6.3.

One can prove a few more facts using the Res-Cor sequences. From the Swan spectral sequence [12] arising from the action $A(z, w)=(\zeta z, w), B(z, w)=(z, \zeta w)$, $\zeta=e^{2 \pi / p}$, of $G$ on $S^{1} \times S^{1}$, one gets various sequences and estimates, none of which seem any good, except as a means of checking the results received via the spectral sequences of group extensions. To these we now turn.

6.3. Set $D=\langle C\rangle, G / D=L \approx Z_{p} \oplus Z_{p}$. We will study the spectral sequence of the extension $1 \rightarrow D \rightarrow G \rightarrow L \rightarrow 1$, following (more or less) the footsteps of Evens. We will refer to this as the first spectral sequence. $E_{2}^{i, j} \cong H^{i}\left(L, H^{j}(D, Z)\right.$ ). As $D=Z(G)$, the action of $L$ on $H^{j}(D, Z)$ is trivial.

Let $\gamma_{0} \in H^{2}(D, Z)$ be a maximal generator, and let $\gamma \in E_{2}^{0,2}$ correspond to $\gamma_{0}$. [For clarity, we will write $Z_{p} \oplus Z_{p}$ for $L$, and $Z_{p}$ for $D$.] From an additive point of view,

$$
E_{2}^{*, 2 j} \cong H^{*}\left(Z_{p} \oplus Z_{p}, Z_{p}\right) \approx H^{*}\left(Z_{p}, Z_{p}\right) \otimes H^{*}\left(Z_{p}, Z_{p}\right),
$$

by Künneth. However, the multiplication on the right-hand side differs from that in the spectral sequence. In fact, it induces a "horizontal" multiplication $\circ: E_{2}^{i, 2 j} \otimes E_{2}^{k, 2 j} \rightarrow E_{2}^{i+k, 2 j}$, each $j>0$. 
LeMmA 6.4. Cup-product $\gamma: H^{i}\left(L, H^{j}(D, Z)\right) \stackrel{\approx}{\rightarrow} H^{i}\left(L, H^{j+2}(D, Z)\right)$ is induced by cup-product $\gamma_{0}: H^{j}(D, Z) \stackrel{\mathcal{Z}}{\rightarrow} H^{j+2}(D, Z)$.

LEMMA 6.5. If $\eta \in E_{2}^{i, 2 j}, \xi \in E_{2}^{k, 2 j}$, then $\eta \xi=\gamma^{j} \cdot(\eta \circ \xi)$. In other words,

$$
H^{*}\left(L, H^{*}(D, Z)\right) \cong H^{*}\left(L, Z_{p}\right) \otimes H^{*}(D, Z),
$$

as rings, for $j>0$.

LEMMA 6.6. $\gamma: E_{2}^{i, j} \rightarrow E_{2}^{i, j+2}$ is mono, $j \geqq 0$, and iso, $j>0$.

The proofs of these lemmas are routine, so we omit them (see [3, Chapter XII, $\S 6])$.

We may now calculate some of the structure of $E_{2}$. Let $H^{*}(L, Z)=P[\alpha, \beta] \otimes E(\delta)$, $\alpha, \beta$ as usual, $\alpha, \beta \in E_{2}^{2,0}$. Let $\mu, \nu \in E_{2}^{1,2}$ be independent generators, and $\chi=\mu \circ \nu$ $\in E_{2}^{2,2}$. As $H^{2}(G, Z)=Z \alpha \oplus Z \beta$, we must have $d_{3}(\gamma)=s \delta, s \in Z_{p}^{*}$. By Proposition $6.1, \alpha^{2}, \alpha \beta, \beta^{2}$ are linearly independent, hence $d_{3} \mu=d_{3} \nu=0$. Thus in degree 3 , only $\mu$ and $\nu$ survive, whence $H^{3}(G, Z)=Z \mu \oplus Z \nu$. Of course, $p \mu=p \nu=0$. [This fills the gap in the proof of Proposition 6.3.] $d_{2}\left(\gamma^{2}\right)=2 s \gamma \delta \neq 0, d_{2}(\gamma \alpha)=\delta \alpha \neq 0, d_{2}(\gamma \beta)=\delta \beta \neq 0$. By Proposition 6.3, $H^{4}(G, Z)$ has order $p^{4}$, hence $d_{3} \chi=0$, and $H^{4}(G, Z)=Z \alpha^{2}$ $\oplus Z \alpha \beta \oplus Z \beta^{2} \oplus Z_{\chi}$.

Lemma 6.7. For suitable choice of $\mu, \nu, \delta, \gamma \delta=\alpha \mu-\beta \nu$ in $E_{2}$.

Proof. In the doubly periodic resolution for $L$ given in $\S 3$, we take cocycles

$$
\begin{aligned}
& \delta: a_{03} \rightarrow 0, a_{12} \rightarrow-1, a_{21} \rightarrow 1, a_{30} \rightarrow 0, \\
& \mu: a_{01} \rightarrow \gamma_{0}, a_{10} \rightarrow 0, \\
& \nu: a_{10} \rightarrow \gamma_{0}, a_{01} \rightarrow 0,
\end{aligned}
$$

representing classes $\delta \in H^{3}\left(Z_{p} \oplus Z_{p}, Z\right), \quad \mu, \nu \in H^{1}\left(Z_{p} \oplus Z_{p}, H^{2}\left(Z_{p}, Z\right)\right)$. Thus $\gamma \delta \in H^{3}\left(Z_{p} \oplus Z_{p}, H^{2}\left(Z_{p}, Z\right)\right)$ is represented by $f_{\gamma_{0}} \circ \delta$, where $f_{\gamma_{0}}: Z \rightarrow H^{2}\left(Z_{p}, Z\right)$ map $1 \rightarrow \gamma_{0}$ (i.e. $f_{\gamma_{0}}=$ cup-product with $\gamma_{0}$ ). As for $\alpha \mu-\beta \nu$, by Lemma 3.4, cupproduct with the periodicity cocycles $\alpha, \beta$ (here $\alpha: a_{10} \rightarrow 1, a_{01} \rightarrow 0, \beta: a_{01} \rightarrow 1$, $a_{10} \rightarrow 0$ ) is just a shift two units east (resp. north). Thus by inspection, $\alpha \mu-\beta \nu$ is represented by the same cocycle that represents $\gamma \delta$. Q.E.D.

REMARK 1. One easily checks that the above $\alpha, \beta$ correspond to the inflations (previously called $\alpha, \beta)$ of the standard generators of $H^{2}(\langle A\rangle, Z)$, resp. $H^{2}(\langle B\rangle, Z)$. We tacitly assumed this in the proof.

LEMMA 6.8. $\delta \mu=-\beta \chi, \delta \nu=-\alpha \chi, \delta \chi=0, \mu \chi=\nu \chi=0, \mu^{2}=\nu^{2}=\chi^{2}=0$.

Proof. The projection $J: Z \rightarrow Z_{p}$ induces

$$
J_{*}: H^{i}\left(Z_{p} \oplus Z_{p}, H^{j}\left(Z_{p}, Z\right)\right) \rightarrow H^{i}\left(Z_{p} \oplus Z_{p}, H^{j}\left(Z_{p}, Z_{p}\right)\right) .
$$

$J_{*}$ is mono, $j \geqq 0$, and iso, $j$ even, $>0$. As in $\S 3$, we have $H^{*}\left(Z_{p} \oplus Z_{p}, Z_{p}\right) \approx H^{*}\left(Z_{p}, Z_{p}\right) \otimes H^{*}\left(Z_{p}, Z_{p}\right)=E\left(u_{1}\right) \otimes P\left(v_{1}\right) \otimes E\left(u_{2}\right) \otimes P\left(v_{2}\right)$, with $\Delta u_{i}=v_{i}, i=1,2$. 
We take as representative cocycles $u_{1}: a_{10} \rightarrow 1, a_{01} \rightarrow 0, u_{2}: a_{01} \rightarrow 1, a_{10} \rightarrow 0$, $v_{1}: a_{20} \rightarrow 1, a_{11} \rightarrow 0, a_{02} \rightarrow 0, v_{2}: a_{02} \rightarrow 1, a_{11} \rightarrow 0, a_{20} \rightarrow 0$. Let $\bar{\gamma}=J_{*}(\gamma)$. Note that $J_{*}$ preserves products. We now observe $J_{*}(\delta)=v_{1} u_{2}-v_{1} u_{1} \quad\left(=\Delta\left(u_{1} u_{2}\right)\right)$, $J_{*}(\alpha)=v_{1}, J_{*}(\beta)=v_{2}, J_{*}(\mu)=\bar{\gamma} u_{2}, J_{*}(\nu)=\bar{\gamma} u_{1}, J_{*}(\alpha)=\bar{\gamma} u_{1} u_{2}$. Thus

$$
J_{*}(\delta \mu)=\left(v_{1} u_{2}-v_{2} u_{1}\right) \bar{\gamma} u_{2}=-v_{2} u_{1} u_{2} \bar{\gamma}=-J_{*}(\beta \chi) .
$$

$J_{*}$ mono implies that $\delta \mu=-\beta \chi$. Likewise, $\delta \nu=-\alpha \chi$. The other relations follow in the same way. Q.E.D.

We observe also that $\mu \nu=\gamma \chi$ (by Lemma 6.5). Let us now insist that $\gamma_{0} \in H^{2}(D, Z)$ be the maximal generator given by $\gamma_{0}: C \rightarrow 1 / p$. As in the proof of Proposition 6.3, if $H=\langle B, C\rangle, H^{*}(H, Z)=P[\beta, \gamma] \otimes E(\mu), k=$ automorphism induced by $A$, then $k \beta=\beta, k \gamma=\gamma+\beta, \operatorname{Res}_{D}(\gamma)=\gamma_{0}, \operatorname{Res}_{D}(\beta)=0$.

LeMma 6.9. $\gamma^{p} \in E_{2}^{0,2 p}$ is a universal cycle. [N.B. There are two distinct $\gamma$ 's, one $\gamma \in E_{2}^{0,2}$, the other $\gamma \in H^{2}(H, Z)$.]

Proof. By the double coset formula for $\mathscr{N}$ [5], we see that

$$
\operatorname{Res}_{H} \mathscr{N}(\gamma)=\prod_{i=0}^{p-1}(\gamma-i \beta)=\gamma^{p}-\gamma \beta^{p-1}
$$

Hence $\operatorname{Res}_{D} \mathscr{N}(\gamma)=\operatorname{Res}_{D} \gamma^{p}=\gamma_{0}^{p}$, i.e. $=\gamma^{p}$, via $H^{2 p}(D, Z) \approx E_{2}^{0,2 p}$. By Corollary II, $\S 1, \gamma^{p}$ is a universal cycle. Q.E.D.

Lemma 6.6 now shows that $\gamma^{p}: E_{2}^{i, j} \rightarrow E_{2}^{i, j+2 p}$ is an isomorphism for $j>0$.

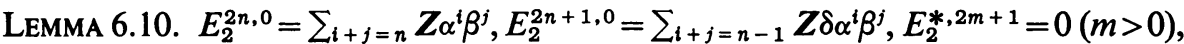

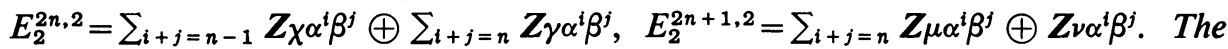
other terms are given by periodicity $E_{2}^{*, 2} \approx^{\gamma} E_{2}^{*, 4} \approx \cdots$.

Proof. By inspection, in view of our previous results. Q.E.D.

Clearly $E_{2}=E_{3}$, as odd rows are zero. We have seen that $\alpha, \beta, \mu, \nu, \chi, \gamma^{p}$ are universal cycles. To find $E_{4}$, we must get $d_{3}$. Recall that $d_{3}(\gamma)=s \delta, s \not 0(p)$.

LEMMA 6.11. $E_{4}^{2 n, 2 m}=0$ if $m \neq \equiv(p), m>0, n \neq 1, E_{4}^{2,2 n}=Z_{\chi \gamma^{m-1}}, m>0, E_{4}^{2 n, 0}$

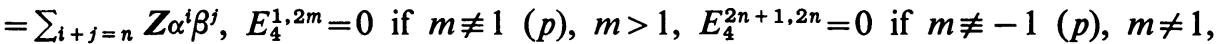
$n>0, \quad E_{4}^{2 n+1,2(p-1)}=\sum_{i+j=n-1} Z \alpha^{i} \beta^{j} \gamma^{p-1} \delta, \quad E_{4}^{2 n+1,2}=\sum_{i+j=n} Z \alpha^{i} \beta^{j} \mu \oplus \boldsymbol{Z}^{i} \beta^{j} v \oplus$ $\sum_{i+j=n-1} Z \alpha^{i} \beta^{j}(\alpha \mu-\beta v)$. The other terms are given by periodicity $E_{4}^{*, j} \approx^{\gamma p} E_{4}^{*, j+2 p}$, $j>0$.

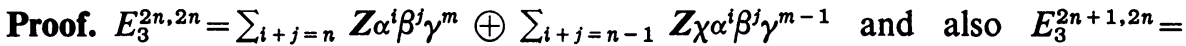

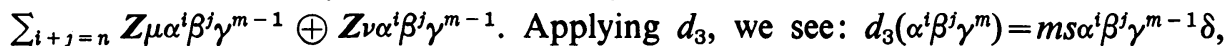
$d_{3}\left(\chi^{i} \beta^{j} \gamma^{m-1}\right)=(m-1) s \alpha^{i} \beta^{j} \gamma^{m-2} \chi^{\delta}=0$, as $\chi^{\delta}=0$.

$$
d_{3}\left(\mu \alpha^{i} \beta^{j} \gamma^{m-1}\right)=(m-1) s \mu \alpha^{i} \beta^{j} \gamma^{m-2} \delta=(m-1) s \alpha^{i} \beta^{j+1} \chi \gamma^{m-2},
$$

as $\mu \delta=-\delta \mu=\beta \chi$. Likewise, $d_{3}\left(\nu \alpha^{i} \beta^{j} \gamma^{m-1}\right)=(m-1) s \alpha^{i+1} \beta^{j} \chi \gamma^{m-2}$. We deduce: The 
terms $\alpha^{i} \beta^{j} \gamma^{m}$ perish if $m \not \equiv 0(p)$, as do the $\chi^{i} \beta^{j} \gamma^{m-1}$ if $m \neq \equiv(p)$ and $i>0$ or $j>0$. The $\mu \alpha^{i} \beta^{j} \gamma^{m-1}$ and $\alpha^{i} \beta^{j} \gamma^{m-1}$ perish for $m \neq \equiv 1(p)$, but

$$
\mu \alpha^{i} \beta^{j} \gamma^{m-1}-\nu \alpha^{i-1} \beta^{j+1} \gamma^{m-1}=\alpha^{i-1} \beta^{j} \gamma^{m-1}(\alpha \mu-\beta \nu)=\alpha^{i-1} \beta^{j} \gamma^{m} \delta
$$

goes to zero under $d_{3}\left(\delta^{2}=0\right) . d_{3}\left(\gamma^{m+1} \delta\right)=(m+1) s \gamma^{m} \delta$, so if $m \not \equiv-1(p)$, the $\alpha^{i-1} \beta^{j} \gamma^{m} \delta$ perish because they are boundaries. Putting these facts together and examining a few special cases, gives the result. Q.E.D.

Corollary 6.12. $\alpha \mu=\beta \nu$ in $H^{*}(G, Z)$, whence $\alpha \mu \nu=\beta \mu \nu=0$.

Proof. $d_{3}\left(\gamma^{2}\right)=2 \gamma \delta=2(\alpha \mu-\beta)$, so $\alpha \mu=\beta$ in $E_{4}$. But $E_{4}^{i, j}=0, i+j=5,(i, j) \neq(3,2)$. The first statement is now clear. As $\mu^{2}=\nu^{2}=0 H^{*}(G, Z), \mu$ and $\nu$ being of odd degree, the second assertion follows. Done.

We have actually shown that $H^{5}(G, Z)=Z \alpha \mu \oplus Z \alpha \nu \oplus Z \beta \mu$, so that $\alpha \mu=\beta \nu$ is the only relation in dimension 5. Let $a, \lambda$ be the automorphisms of $G$ given by $a: A \rightarrow B, B \rightarrow A, \lambda: B \rightarrow B, A \rightarrow A B$, respectively. $a^{2}=1, \lambda^{p}=1$. Clearly

$$
\alpha^{a}=\beta, \quad \beta^{a}=\alpha ; \quad \alpha^{\lambda}=\alpha, \quad \beta^{\lambda}=\beta+\alpha .
$$

Only the second part needs checking. $\alpha: A \rightarrow 1 / p, B \rightarrow 0, \beta: A \rightarrow 0, B \rightarrow 1 / p$. By the definition of $\S 1, \alpha^{\lambda}=\alpha \circ \lambda, \beta^{\lambda}=\beta \circ \lambda$. So $\alpha^{\lambda}(A)=\alpha(A B)=1 / p . \alpha^{\lambda}(B)=\alpha(B)=0$. $\beta^{\lambda}(A)=\beta(A B)=1 / p$. $\beta^{\lambda}(B)=\beta(B)=1 / p$. Done.

$a$ and $\lambda$ act on $H^{3}(G, Z)$. Suppose $\mu^{a}=d \mu+e \nu, \nu^{a}=f \mu+g \nu . \alpha \mu=\beta \nu$ implies $\beta \mu^{a}=\alpha \nu^{a}$, or $\beta(d \mu+e \nu)=\alpha(f \mu+g \nu)$, or $d \beta \mu=(f-e) \alpha \mu+g \alpha \nu$, implying $d=g=0$, $f=e . \mu^{a}=e v . a^{2}=1$ so $e^{2}=1$, hence $e= \pm 1$ (everything is $\bmod p$, of course).

The diagram (obtained from the spectral sequence) commutes:

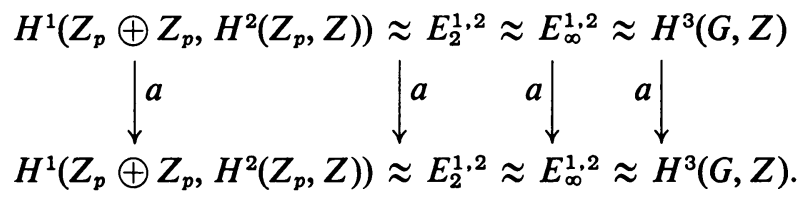

Hence by the definition of $\mu, \nu$ we see that $\mu^{a}=-\nu$. (Since $c^{a}=c^{-1}$ implies that $a: H^{2}\left(Z_{p}, Z\right) \rightarrow H^{2}\left(Z_{p}, Z\right)$ is multiplication by -1 .) Thus

$$
\mu^{a}=-\nu, \quad \nu^{a}=-\mu .
$$

A similar argument yields

$$
\mu^{\lambda}=\mu+\nu, \quad \nu^{\lambda}=\nu .
$$

Let $H_{t}=\left\langle A B^{t}, C\right\rangle . H=H_{p}=\langle B, C\rangle$.

Lemma 6.16. $\operatorname{Res}_{H} \mu \neq 0, \operatorname{Res}_{H} \nu=0$.

Proof. Observe $\lambda(C)=C$. Thus $\lambda=$ identity on $H$. Thus

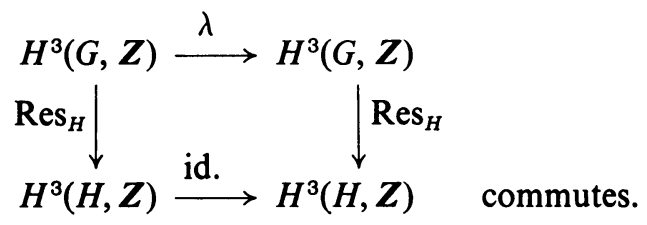


We saw in the proof of Proposition 6.3 that $\operatorname{Res}_{H}$ is onto. Let $d \mu+e \nu \neq 0$ be an element such that $\operatorname{Res}_{H}(d \mu+e \nu)=0$. Apply $\lambda$, get $\operatorname{Res}_{H}(d(\mu+\nu)+e \nu)=0$. Subtract, then $d \operatorname{Res}_{H}(\nu)=0$. If $d \neq 0$, then $\operatorname{Res}_{H} \nu=0$. If $d=0$, then $e \neq 0$, and so $\operatorname{Res}_{H} \nu=0$ again. Thus $\operatorname{Res}_{H} \nu=0$. As $\operatorname{Res}_{H} \neq 0$, so $\operatorname{Res}_{H} \mu \neq 0$. Q.E.D.

Observe that $H_{t}=H_{0}^{\lambda t}, H_{0}=H^{a}$. Applying $a$ to Lemma 6.16 gives $\operatorname{Res}_{H_{0}} \nu \neq 0$. $\operatorname{Res}_{H_{0}} \mu=0$.

Claim. $\operatorname{Res}_{H_{t}}(\mu) \neq 0$ if and only if $t \neq 0$. Indeed, apply $\lambda^{t}$ to $\operatorname{Res}_{H_{0}}(\mu)=0$. Get $\operatorname{Res}_{H_{t}}(\mu+t \nu)=0(0<t<p)$. If $\operatorname{Res}_{H_{t}}(\mu)=0$, then $\operatorname{Res}_{H_{t}}(\nu)=0$, implying $\operatorname{Res}_{H_{t}}=0$. Contradiction. Hence $\operatorname{Res}_{H_{t}}(\mu) \neq 0$ if $0<t<p$. By Lemma 6.16 this holds also for $t=p$. Finally, observe that in $H^{*}\left(H_{t}, Z\right)$, no element of even degree is a zero-divisor. Thus, if $\xi \in H^{*}(G, Z)$, of even degree, $\operatorname{Res}_{H_{t}}(\xi)=0$ if and only if $\operatorname{Res}_{H_{t}}(\xi \mu)=0$, for $t \neq 0$.

Proposition 6.17. The elements $\alpha^{i} \mu, \alpha^{i-1} \beta \mu, \ldots, \beta^{i} \mu, \alpha^{2} \nu$ in $H^{2 i+3}(G, Z)$ are linearly independent for $0 \leqq i<p$.

Proof. Exactly as in Proposition 6.1. Obtain $a_{t} \in H^{2}(G, Z), \operatorname{Res}_{H_{j}}\left(a_{t}\right)=0$ if and only if $t=j$. Let $I \subset\{1, \ldots, p\}$ consist of $i$ elements. Form $a_{I}=\prod_{i \in I} a_{i}$. Then we see that $\left\{A_{I} \mu\right\}$ contains at least $i+1$ linearly independent elements. As the $A_{I}$ lie in the span of $\left\{\alpha^{i} \mu, \ldots, \beta^{i} \mu\right\}$, the latter set of $i+1$ elements is linearly independent. As for $\alpha^{i} \nu$, we saw that $\operatorname{Res}_{H_{0}}\left(\alpha^{i} \nu\right) \neq 0, \operatorname{Res}_{H_{0}}\left(\alpha^{k} \beta^{l} \mu\right)=0$. Hence the enlarged set $\left\{\alpha^{i} \mu, \ldots, \beta^{i} \mu, \alpha^{i} \nu\right\}$ is still independent. Q.E.D.

Lemma 6.18. $\gamma^{i} \chi \in E_{4}^{2,2 i+2}$ survives to $E_{\infty}$ for $0 \leqq i<p-1$.

Proof. As the fibre terms are zero, or universal cycles, need only show $\gamma^{i} \chi$ are universal cycles. $d_{2 i+1}: E_{4}^{2,2 i+2} \rightarrow E_{4}^{2(i+1)+1,2}$ is the only possibly nonzero differential.

As $E_{4}^{2(i+1)+1,2}$ is generated by the $\alpha^{i+1} \mu, \ldots, \beta^{i+1} \mu, \alpha^{i+1} \nu$ and $i+1 \leqq p$, Proposition 6.17 implies that $d_{2 i+1}\left(\gamma^{i} \chi\right)=0$. Q.E.D.

Thus, in degrees $\leqq 2 p, E_{4}=E_{\infty}$. Inspection reveals:

$$
\alpha_{2 i}=p^{i+2}, \quad 2 \leqq i<p ; \quad \alpha_{2 i+1}=p^{i+1}, \quad 1 \leqq i<p-1 ; \quad \alpha_{2 p}=p^{p+3} .
$$

LEMMA 6.20. $\alpha_{2 p+1}=p^{p+1}, \alpha_{2 p+2}=p^{p+3}, \alpha \beta^{p}=\beta \alpha^{p}, \alpha^{p} \mu=\beta^{p} \nu$.

Proof. As $\alpha_{1}=1, \alpha_{2}=p^{2}$, inequality (6.2) gives $\alpha_{2 p+1} \leqq p^{p+1}, \alpha_{2 p+2} \leqq p^{p+3}$. The $p+1$ elements $\alpha^{p-1} \mu, \ldots, \beta^{p-1} \mu, \alpha^{p-1} \nu \in E_{4}^{2 p-1,2}$ are linearly independent and survive to $E_{\infty}$ (Proposition 6.17). This implies $\alpha_{2 p+1}=p^{p+1}$. This shows also that $d_{2 p-1}\left(\gamma^{p-1} \delta\right) \neq 0$. In degree $2 p+2$, there are independent generators $\gamma^{p} \alpha, \gamma^{p} \beta, \gamma^{p-1} \chi$ $\in E_{4}^{2,2 p}$, and the $\alpha^{p+1}, \ldots, \beta^{p+1}$, in $E_{4}^{2 p+2,0}$. As $d_{2 p-1}\left(\gamma^{p-1} \delta\right) \neq 0$, there is precisely one nontrivial relation, $f(\alpha, \beta)=0$. Thus, as $\gamma^{p} \alpha, \gamma^{p} \beta$ survive to $E_{\infty}$, we get $\alpha_{2 p+2}$ $\geqq p^{p+3}$, implying that $\alpha_{2 p+2}=p^{p+3}$. Thus $\gamma^{p-1} \chi$ perishes, so $d_{2 p-1}\left(\gamma^{p-1} \chi\right) \neq 0$. This says that there is precisely one relation among the elements $\alpha^{p} \mu, \alpha^{p-1} \beta \mu, \ldots$, 
$\beta^{p} \mu, \alpha^{p} \nu$. To find the relations, we apply the automorphisms $a$ and $\lambda$. Thus, $f(\alpha, \beta)=0$ implies $f(\beta, \alpha)=0$, and $f(\alpha, \alpha+\beta)=0$.

Let

$$
\begin{aligned}
f(\alpha, \beta) & =\sum_{i=0}^{p+1} c_{i} \alpha^{i} \beta^{p+1-i} \\
f(\alpha, \alpha+\beta) & =\sum_{i=0}^{p+1} c_{i} \alpha^{i}(\alpha+\beta)^{p+1-i}=\sum_{0 \leqq i \leqq p+1 ; 0 \leqq j \leqq p+1-i} c_{i}\left(\begin{array}{c}
p+1-i \\
j
\end{array}\right) \alpha^{j+i} \beta^{p+1-j-i} \\
& =\sum_{i^{\prime}=0}^{p+1} \sum_{0 \leqq i \leqq i^{\prime}} c_{i}\left(\begin{array}{c}
p+1-i \\
i^{\prime}-i
\end{array}\right) \alpha^{i^{\prime}} \beta^{p+1-i^{\prime}}
\end{aligned}
$$

By uniqueness of the relation, $f(X, X+Y)=e f(X, Y)$, some integer $e$. Iteration $p$ times yields $e^{p}=1$, whence $e=1$ (everything is $\bmod p$ ). Thus, we may equate coefficients:

$$
c_{0}\left(\begin{array}{c}
p+1 \\
r
\end{array}\right)+c_{1}\left(\begin{array}{c}
p \\
r-1
\end{array}\right)+c_{2}\left(\begin{array}{c}
p-1 \\
r-2
\end{array}\right)+\cdots+c_{r}\left(\begin{array}{c}
p-r+1 \\
0
\end{array}\right)=c_{r}
$$

$(0 \leqq r \leqq p+1)$. This plus symmetry implies $c_{0}=c_{2}=\cdots=c_{p-1}=c_{p+1}=0$, and $c_{1}+c_{p}=0$. We may take $c_{1}=1$. Thus $f(\alpha, \beta)=\alpha \beta^{p}-\beta \alpha^{p}$. The relation $\alpha^{p} \mu=\beta^{p} \nu$ is proved similarly. Q.E.D.

We now have the $E_{\infty}$ term $\left(=E_{2 p}\right)$. Inspection shows that

$$
\alpha_{j+2 p}=p^{p+1} \alpha_{j}, \quad j>0,
$$

as previously asserted. The elements $\chi, \gamma \chi, \ldots, \gamma^{p-2} \chi$ are in $E_{\infty}$. How do they multiply? To settle this we use the Res-Cor sequences associated with $1 \rightarrow H \rightarrow G$ $\rightarrow\langle A\rangle \rightarrow 1$. We know that $H^{*}(H, Z)=P[\beta, \gamma] \otimes E(\mu) . \operatorname{Res}_{H} \beta=\beta, \operatorname{Res}_{H} \mu=\mu$, and $\operatorname{Res}_{H} \mathscr{N}(\gamma)=\gamma^{p}-\gamma \beta^{p-1}$ (proof of Lemma 6.9). Let $\zeta=\gamma^{p}-\gamma \beta^{p-1}$. As $k \beta=\beta$, $k \gamma=\gamma+\beta$, we find: $H^{2 i}(H, Z)^{k}=Z \beta^{i}, 0<i<p . H^{2 i+1}(H, Z)^{k}=Z \mu \beta^{i-1}, 0<i<p-1$, $H^{2 p}(H, Z)^{k}=Z \beta^{p} \oplus Z \zeta . \quad H^{2 i}(H, Z)_{k}=Z \gamma^{i}, \quad 1 \leqq i<p, \quad H^{2 i+1}(H, Z)_{k}=Z \mu \gamma^{i-1}$, $0<i \leqq p$.

Lemma 6.22. Cor $\gamma=0$, Cor $\gamma^{i} \neq 0,1 \leqq i \leqq p$.

Proof. First observe Cor: $H^{2 i+1}(H, Z) \rightarrow H^{2 i+1}(G, Z)$ is $0,0 \leqq i<p$. Indeed, the formula $\operatorname{Cor}(\operatorname{Res} a \cdot b)=a \cdot \operatorname{Cor} b$ [3, Chapter XII, §8], Res $\alpha=0$, imply $\alpha$ Cor $\xi=0$ for $\xi \in H^{*}(H, Z)$. If $\xi \in H^{2 i+1}(H, Z)$, then Cor $\xi \in H^{2 i+1}(G, Z)$ lies in the span of $\alpha^{i} \mu, \ldots, \beta^{i} \mu, \alpha^{i} \nu$. As $\alpha$ annihilates no nonzero combination of these (Proposition 6.17), so $\alpha$ Cor $\xi=0$ implies Cor $\xi=0 . H^{2 i}(H, Z)^{k}=Z \beta^{i}, H^{2 i+1}(H, Z)^{k}$ $=Z \mu \beta^{i-1}$, show that the image of $\operatorname{Res}: H^{21}(G, Z) \rightarrow H^{21}(H, Z)$ has order $p$ $(1 \leqq i<p)$, and order $p^{2}$ if $i=p$, since $H^{2 p}(H, Z)^{k}=Z \beta^{p} \oplus Z \zeta$. By the Res-Cor sequences:

$$
\begin{aligned}
& 0 \rightarrow Z_{p} \rightarrow H^{2 i}(H, Z) \rightarrow H^{2 i}(G, X) \rightarrow H^{2 i+1}(G, Z) \rightarrow Z_{p} \rightarrow 0, \text { exact }(1 \leqq i<p), \\
& 0 \rightarrow Z_{p} \oplus Z_{p} \rightarrow H^{2 p}(H, Z) \rightarrow H^{2 p}(G, X) \rightarrow H^{2 p+1}(G, Z) \rightarrow Z_{p} \rightarrow 0, \text { exact. }
\end{aligned}
$$


$\left|H^{2 i}(H, Z)\right|=p^{i+1}$, so by (6.19) and Lemma 6.20 , we get: $\left|H^{2 i}(G, X)\right|=p^{2 i}$, $2 \leqq i<p,\left|H^{2 p}(G, X)\right|=p^{2 p-1}$. If $\mathrm{Cor}_{2 i}=0,2 \leqq i<p$, then

$$
0 \rightarrow H^{2 i-1}(G, Z) \rightarrow H^{2 i}(G, X) \rightarrow H^{2 i}(H, Z) \rightarrow 0
$$

were exact. This implies $\left|H^{2 i}(G, X)\right|=p^{i} \cdot p^{i+1}=p^{2 i+1}$, a contradiction. Therefore $\operatorname{Cor}_{2 i} \neq 0$, and hence $\operatorname{Cor}\left(\gamma^{i}\right) \neq 0,2 \leqq i<p$.

Res Cor $\gamma^{p-1}=N_{k} \gamma^{p-1}=-\beta^{p-1}$ implies Res $\left(\operatorname{Cor} \beta \gamma^{p-1}\right)=-\beta^{p} \neq 0$. Thus if Cor $\gamma^{p}=0$, then

$$
0 \rightarrow H^{2 p-1}(G, Z) \rightarrow H^{2 p}(G, X) \rightarrow H^{2 p}(H, Z) \rightarrow Z_{p} \rightarrow 0
$$

were exact. This implies $p\left|H^{2 p}(G, X)\right|=p^{p} \cdot p^{p+1}=p^{2 p+1}$, or $\left|H^{2 p}(G, X)\right|=p^{2 p}$. A contradiction. Hence Cor $\gamma^{p} \neq 0$. As for Cor $\gamma=0$, we may check this directly using the formula in $\S 1$. Q.E.D.

COROLlaRy 6.23. $\mathcal{N}(\gamma)$ has order $p^{2}$.

Proof \#1. As $\operatorname{Res}_{H} \mathcal{N}(\gamma)=\gamma^{p}-\gamma \beta^{p-1}$, apply Cor, and note Cor $\gamma=0$. Get $p \mathscr{N}(\gamma)=$ Cor $\gamma^{p} \neq 0$, by previous result. Done.

Proof \#2. Glance back at (6.1)-(6.1) $)^{m}$. Clearly Cor: $\hat{H}^{0}(H, Z) \rightarrow \hat{H}^{0}(G, Z)$ has cokernel cyclic of order $p^{2}$. That is, $H^{1}(G, X) \approx Z_{p^{2}}$. We see that

$$
\begin{aligned}
\left|H^{2 p}(G, Z)\right| & \leqq p^{p}\left|H^{2 p-1}(G, U)\right|=p^{p}\left|H^{p}(G, W)\right| \\
& \leqq p^{p}\left|H^{p-1}(G, V)\right|=p^{p}\left|H^{2}(G, Y)\right| \leqq p^{p+1}\left|H^{1}(G, X)\right|=p^{p+3} .
\end{aligned}
$$

But we know that $\alpha_{2 p}=p^{p+3}$, whence, equality holds at every stage. In particular, $H^{p}(G, V) \stackrel{\text { ep } 1}{\longrightarrow} H^{p}(G, W)$ is actually an isomorphism. So

$$
\begin{aligned}
Z_{p^{2}} & =H^{1}(G, X) \stackrel{\text { mono }}{\longrightarrow} H^{2}(G, Y) \approx H^{p-1}(G, V) \approx H^{p}(G, W) \\
& \approx H^{2 p-1}(G, U) \rightarrow H^{2 p}(G, Z) .
\end{aligned}
$$

Thus $H^{2 p}(G, Z)$ contains an element of order $p^{2}$. Now the spectral sequence, Res Cor $\gamma^{p}=N_{k} \gamma^{p}=0$, and Cor $\gamma^{p} \neq 0$, plus $p$ Cor $\gamma^{p}=0$, and $H^{2 p}(G, Z)=Z \mathscr{N}(\gamma)$ $\oplus \boldsymbol{Z} \operatorname{Cor} \gamma^{p} \oplus \sum_{i+j=p} \boldsymbol{Z} \alpha^{i} \beta^{j}$, show that $\mathcal{N}(\gamma)$ is of order $p^{2}$. Q.E.D.

Remark 1. $\alpha$ Cor $\gamma^{p}=0$, Res Cor $\gamma^{p}=0$ are what tell us that Cor $\gamma^{p} \notin \sum Z \alpha^{i} \beta^{j}$. For if so, then applying Res shows that Cor $\gamma^{p}$ lies in $\boldsymbol{Z} \alpha \beta^{p-1} \oplus \cdots \oplus \boldsymbol{Z} \alpha^{p}$. As $\alpha \beta^{p}=\alpha^{p} \beta$ is the only relation in degree $2 p+2$, we see that $\alpha$ Cor $\gamma^{p}=0$ implies Cor $\gamma^{p}=0$. Contradiction.

Let $\chi_{i}=\operatorname{Cor} \gamma^{i+1}, 1 \leqq i<p-2$. Note $\chi_{i}^{\lambda}=\chi_{i}, \alpha \chi_{i}=0$. As in Remark 1, we see that $\chi_{i}$ is not a polynomial in $\alpha$ and $\beta$. The spectral sequence implies that $H^{2 i+2}(G, Z)$ $=Z_{\chi_{i}} \oplus \sum_{k+l=i+1} Z_{\alpha^{k}} \beta^{l},(1 \leqq i<p-2)$.

LEMmA 6.24. $\chi_{i} \chi_{j}=0,1 \leqq i, j<p-2, \alpha \chi_{i}=\beta \chi_{i}=\mu \chi_{i}=\nu \chi_{i}=0, \quad \chi_{i}^{\lambda}=\chi_{i}, \quad \chi_{i}^{a}=\varepsilon_{i} \chi_{i}$, $\varepsilon_{i}= \pm 1 . \operatorname{Res}_{N}\left(\chi_{i}\right)=0$, for all proper subgroups $N$ of $G$. 
Proof. The fact that $\operatorname{Res}_{H} \chi_{i}=N_{k} \chi^{i+1}=0$ is easily checked. The formula Cor $(\operatorname{Res} a \cdot b)=a \cdot$ Cor $b$ now implies $\chi_{i} \chi_{j}=0, \quad \alpha \chi_{i}=0, \quad v \chi_{i}=0 . \quad\left(\alpha \chi_{i}\right)^{a}=\beta \chi_{i}^{a}=0$, $\left(\nu \chi_{i}\right)^{a}=\mu \chi_{i}^{a}=0$. So we need specify $\chi_{i}^{a}$. Let $\chi_{i}^{a}=\varepsilon_{i} \chi_{i}+f_{i}(\alpha, \beta)$, implying $\chi_{i}=\varepsilon_{i}^{2} \chi_{i}$ $+\varepsilon_{i} f_{i}(\alpha, \beta)+f_{i}(\beta, \alpha)$ which in turn implies that $\varepsilon_{i}= \pm 1$. $\beta \chi_{i}^{a}=0$ implies $0=\varepsilon_{i} \beta \chi_{i}$ $+\beta f_{i}(\alpha, \beta) . \beta \chi_{i}$ is $\lambda$-invariant, as $\alpha \chi_{i}=0$ and $\chi_{i}^{\lambda}=\chi_{i}$. Hence so is $\beta f_{i}(\alpha, \beta)$. But powers of $\alpha$ are the only $\lambda$-invariant polynomials in degree $<2 p$. Hence $f_{i}(\alpha, \beta)=0$ or $\chi_{i}^{a}=\varepsilon_{i} \chi_{i}$. This proves all but the last assertion.

We may assume $N$ is of index $p$, i.e. $N=\left\langle A^{i} B^{j}, C\right\rangle$, some $i, j$. There exists $f(\alpha, \beta)=$ polynomial in $\alpha, \beta$ such that $\operatorname{Res}_{N} f(\alpha, \beta) \neq 0$ (can take $f$ linear). Thus $\operatorname{Res}_{N} f(\alpha, \beta) \operatorname{Res}_{N} \chi_{i}=\operatorname{Res}_{N}\left(f(\alpha, \beta) \chi_{i}\right)=\operatorname{Res}_{N}(0)=0$. Hence $^{\operatorname{Res}_{N}} \chi_{i}=0$. Q.E.D.

REMARK 2. As $\langle c\rangle=Z(G)$ is characteristic, and $\alpha, \beta$ are inflations, it follows that the subring $Z[\alpha, \beta]$ is Aut $(G)$-invariant. The proof of Proposition 6.1 shows that $\chi_{i}$ is the only element (up to constant multiple) of $H^{2 i+2}(G, Z)$ which has $\operatorname{Res}_{N}=0$ for all proper $N$. Thus $Z_{\chi_{i}}$ is also Aut $(G)$-invariant, as is the decomposition of $H^{2 i+2}(G, Z)$.

If $p>3$, then $\chi_{2}$ is defined, and differs from $\mu \nu$ by a constant multiple (both are $\lambda$-invariant). Here $(\mu \nu)^{a}=\mu^{a} \nu^{a}=\nu \mu=-\mu \nu$, so in this case $\varepsilon_{2}=-1$. We now define $\chi_{p-2}=\operatorname{Cor} \gamma^{p-1}+\beta^{p-1}$. As Res Cor $\gamma^{p-1}=-\beta^{p-1}$ so $\operatorname{Res} \chi_{p-2}=0$,

$$
\chi_{p-2} \in H^{2 p-1}(G, Z) \text {. }
$$

LEMMA 6.25. $\alpha \chi_{p-2}=\alpha \beta^{p-1}, \beta \chi_{p-2}=\beta \alpha^{p-1} \cdot \chi_{p-2}^{a}=\chi_{p-2}, \chi_{p-2}^{\lambda}=\chi_{p-2}+(\beta+\alpha)^{p-1}$ $-\beta^{p-1}, \mu \alpha^{p-1}=\mu \chi_{p-2}, \nu \beta^{p-1}=\nu \chi_{p-2}$.

Proof. $\alpha$ Cor $\gamma^{p-1}=0$, so $\alpha \chi_{p-2}=\alpha \beta^{p-1}\left({ }^{*}\right)$. Likewise, $\nu \chi_{p-2}=\nu \beta^{p-1}$. We check easily that $\chi_{p-2} \neq$ a polynomial in $\alpha, \beta$. Hence

$$
H^{2 p-2}(G, Z)=Z_{\chi p-2} \oplus \sum_{k+l=p-1} Z \alpha^{k} \beta^{l} .
$$

Let $\chi_{p-2}^{a}=\varepsilon \chi_{p-2}+g(\alpha, \beta)$. Apply $a$ to $\left(^{*}\right)$. Get $\beta \chi_{p-2}^{a}=\beta \alpha^{p-1}$, or $\varepsilon \beta \chi_{p-2}+\beta g(\alpha, \beta)$ $=\beta \alpha^{p-1}$. Thus $\beta \chi_{p-2}=\varepsilon^{-1}\left(\beta \alpha^{p-1}-\beta g(\alpha, \beta)\right)=f(\alpha, \beta)$. But $\beta \chi_{p-2}=\beta^{p}+\beta$ Cor $\gamma^{p-1}$ $=\beta^{p}+\operatorname{Cor} \beta \gamma^{p-1}$. As $\operatorname{Cor} \beta \gamma^{p-1}$ is $\lambda$-invariant, we get $f(\alpha, \beta)=\alpha^{p}+f(\alpha, \beta)$. Res $\chi_{p-2}=0$. Hence $f(\alpha, \beta)$ has no $\beta^{p}$-term. A small calculation now gives $f(\alpha, \beta)=\beta \alpha^{p-1}$, i.e. $\beta \chi_{p-2}=\beta \alpha^{p-1}$. A further, smaller calculation proves $\varepsilon=1$. Finally, applying $a$ to $\nu \beta^{p-1}=\nu \chi_{p-2}$ yields $\mu \alpha^{p-1}=\mu \chi_{p-2}$. Q.E.D.

Observe that Lemma 6.25 implies that $\alpha \beta^{p}=\beta \alpha^{p}$, and $\alpha^{p} \mu=\beta^{p} v$. Note also that cup-product with $\mathscr{N}(\gamma)$ is a monomorphism (by the spectral sequence). We can summarize our conclusions in one statement:

THEOREM 6.26. The cohomology ring of

$$
G=\left(A, B: A^{p}=B^{p}=[A, B]^{p}=[A,[A, B]]=[B,[A, B]]=1\right),
$$

for $p$ odd, is as follows: $H^{*}(G, Z)=Z\left[\alpha, \beta, \mu, \nu, \zeta, \chi_{1}, \chi_{2}, \ldots, \chi_{p-2}\right], \operatorname{deg} \alpha=\operatorname{deg} \beta$ $=2, \operatorname{deg} \mu=\operatorname{deg} \nu=3, \operatorname{deg} \zeta=2 p, \operatorname{deg} \chi_{i}=2 i+2$, with relations: (0) $p \alpha=p \beta=p \mu$ 
$=p \nu=p \chi_{i}=p^{2} \zeta=0$, (1) $\alpha \mu=\beta \nu$, (2) $\alpha^{p} \mu=\beta^{p} \nu$, (3) $\mu^{2}=v^{2}=0$, (4) $\chi_{i} \chi_{j}=\alpha \chi_{i}=\beta \chi_{i}$

$=\mu \chi_{i}=\nu \chi_{i}=0,1 \leqq i, j<p-2$, (5) $\chi_{i} \chi_{p-2}=0,1 \leqq i<p-2, \quad \chi_{p-2}^{2}=\alpha^{p-1} \beta^{p-1}$,

$\alpha \chi_{p-2}=\alpha \beta^{p-1}, \beta \chi_{p-2}=\beta \alpha^{p-1}$, (7) $\mu \alpha^{p-1}=\mu \chi_{p-2}, \nu \beta^{p-1}=\nu \chi_{p-2}$, (8) $\alpha \beta^{p}=\beta \alpha^{p}$.

If $p>3$, then $\chi_{2}=d \mu \nu$, for some $d \in Z_{p}^{*}$; if $p=3$, then $p \zeta=e \mu \nu$, some $e \in Z_{p}^{*}$. $a, \lambda$ act as follows:

(i) $\alpha^{a}=\beta, \mu^{a}=-\nu, \chi_{i}^{a}=\varepsilon_{i} \chi_{i}, \varepsilon_{i}= \pm 1, \varepsilon_{p-2}=1, \varepsilon_{2}=-1$ if $p>3$,

(ii) $\alpha^{\lambda}=\alpha, \beta^{\lambda}=\beta+\alpha, \nu^{\lambda}=\mu+\nu, \chi_{i}^{\lambda}=\chi_{i}, 1 \leqq i<p-2, \chi_{p-2}^{\lambda}=\chi_{p-2}+(\beta+\alpha)^{p-1}-\beta^{p-1}$, $\zeta^{\lambda}=\zeta$.

Here $a, \lambda: G \rightarrow G$ are: $a: A \rightarrow B, B \rightarrow A, \lambda: B \rightarrow B, A \rightarrow A B$. If $H=\langle B, C\rangle$ (where $C=[A, B]=B^{-1} A^{-1} B A, \gamma \in H^{2}(H, Z)$ corresponding to $C \rightarrow 1 / p, B \rightarrow 0$ ), we may take $\chi_{i}=\operatorname{Cor} \gamma^{i+1}, 1 \leqq i<p-2, \chi_{p-2}=\operatorname{Cor} \gamma^{p-1}+\beta^{p-1}$, and $\zeta=\mathscr{N}(\gamma)$.

Proof. Almost all done in the lemmas, except for a few easy exercises. Q.E.D.

REMARKS. (1) Of course, the above relations are not mutually independent. Thus, for example, (6) implies (8); (6), (7) imply (2).

COROLlary 6.27 .

$$
\text { exponent } \begin{aligned}
H^{n}(G, Z) & =p & & \text { if } 2 p \nmid n, \\
& =p^{2} & & \text { if } 2 p \mid n .
\end{aligned}
$$

6.4. It is of interest to calculate the spectral sequence of

$$
1 \rightarrow H \rightarrow G \rightarrow\langle A\rangle \rightarrow 1 \quad \text { (split), } H=\langle B, C\rangle .
$$

We will call it the second spectral sequence. Set $\mathscr{A}=H^{*}(H, Z)=P[\beta, \gamma] \otimes E(\mu)$, and $\zeta=\gamma^{p}-\gamma \beta^{p-1}$. As before, $k \beta=\beta, k \gamma=\gamma+\beta, k \mu=\mu$. Note $k \zeta=\zeta$. We will often write $Z_{p}$ for $\langle A\rangle, Z_{p} \oplus Z_{p}$ for $H$, and will identify $E_{2}^{0, j} \cong H^{j}\left(Z_{p} \oplus Z_{p}, Z\right)^{k}$.

LEMMA 6.28 .

$$
\begin{aligned}
E_{2}^{i, j} & \approx Z_{p} \quad i, j>0, \quad j \not 0 \quad(p-1) \quad \text { and even } \\
& \approx 0 \quad i, j>0, j \equiv 0 \quad(p-1) \quad \text { and even }
\end{aligned}
$$

\section{Proof.}

$$
\begin{aligned}
E_{2}^{i, j} \cong H^{i}\left(Z_{p}, H^{j}\left(Z_{p} \oplus Z_{p}, Z\right)\right) & =H^{0}\left(\langle k\rangle, H^{j}\left(Z_{p} \oplus Z_{p}, Z\right)\right), & & i \text { even, }>0, \\
& =H^{-1}\left(\langle k\rangle, H^{j}\left(Z_{p} \oplus Z_{p}, Z\right)\right), & & i \text { odd, }>0 .
\end{aligned}
$$

Thus (2), (3), (4), (5) imply (1), by inspection. Obviously, $\mathscr{A}^{k} \supset P[\beta, \zeta] \otimes E(\mu)$. 
$\xi \beta$ or $\xi \mu$ invariant implies $\xi$ invariant. So if $\xi \in \mathscr{A}^{k}$, we may suppose $\xi=\gamma^{n}$ $+a_{n-1} \gamma^{n-1} \beta+\cdots+a_{0} \beta^{n}$. By Newton's formulas, if $s_{r}=1^{r}+2^{r}+\cdots+(p-1)^{r}$, we have

$$
\begin{gathered}
s_{r} \equiv 0[\text { if } r \not \equiv 0(p-1)] \equiv-1[\text { if } r \equiv 0(p-1)] \\
\xi^{k}=\sum_{i=0}^{n} a_{i}(\gamma+\beta)^{i} \beta^{n-i}\left[a_{n}=-1\right]=\gamma^{n}+\left(a_{n-1}+\left(\begin{array}{c}
n \\
1
\end{array}\right)\right) \gamma^{n-1} \beta+\cdots=\xi
\end{gathered}
$$

so equating coefficients gives $n \equiv 0(p)$. Say, $n=p n_{0}$. Form $\xi-\zeta^{n_{0}}=\beta \xi^{\prime}$. This is invariant, implying $\xi^{\prime}$ is invariant. By induction on degrees, $\xi^{\prime} \in P[\beta, \zeta]$, hence so is $\xi$. This proves (2).

$$
N \gamma^{p}=\sum_{j=0}^{p-1}(\gamma+j \beta)^{n}=\sum_{k=1}^{n}\left(\begin{array}{l}
n \\
k
\end{array}\right) s_{k} \gamma^{n-k} \beta^{k}
$$

For $n<p-1$, this is 0 ; for $n=p-1$, it is $-\beta^{p-1}$. Thus:

$$
\begin{aligned}
N \gamma^{i} & =0, & & 1 \leqq i<p-1, \\
& =-\beta^{p-1}, & & i=p-1 .
\end{aligned}
$$

If $\xi \in \mathscr{A}^{k}$, then $N(\xi \eta)=\xi N(\eta)$. By (6.30), if $\xi \in \mathscr{A}^{k}, N\left(\gamma^{p-1} \xi\right)=-\beta^{p-1} \xi$. Hence $N \mathscr{A} \supset \beta^{p-1} \mathscr{A}^{k}$. To show the reverse inclusion, it clearly suffices to prove: $\beta^{p-1} \mid N \gamma^{n}\left(\right.$ for all $n>0$. For then $N \gamma^{n}=\beta^{p-1} \xi_{n}$, and $N \gamma^{n} \in \mathscr{A}^{k}$ implies $\xi_{n} \in \mathscr{A}^{k}$. If $n<p$, we are done, by (6.30). If $n>p$, then $\gamma^{n}=\gamma^{n-p} \zeta+\beta^{p-1} \gamma^{n-p+1}$, implying $n \gamma^{n}=n\left(\gamma^{n-p}\right) \zeta+\beta^{p-1} N\left(\gamma^{n-p+1}\right)$. ( now follows by induction. This proves (3).

Next, let $\xi \in{ }_{N} \mathscr{A}$, i.e. $N \xi=0$. We can suppose $\xi=\gamma^{n}+a_{1} \gamma^{n-1} \beta+\cdots$. We saw that $\beta^{p-1} \mid N \eta$ for all $\eta$. $0=N \xi=N \gamma^{n}+a_{1} N \gamma^{n-1} \cdot \beta+\cdots$, hence $\beta^{p} \mid N \gamma^{n}$. Write $n=c_{0}$ $+c_{1} p+c_{2} p^{2}+\cdots, 0 \leqq c_{i}<p$. We know that $N \gamma^{n}=C_{n, 1} s_{1} \gamma^{n-1} \beta+C_{n, 2} s_{2} \gamma^{n-2} \beta^{2}+\cdots$ $=-C_{n, p-1} \gamma^{n-p+1} \beta^{p-1}+$ multiple of $\beta^{p}$. $\beta^{p} \mid N \gamma^{n}$ implies $C_{n, p-1}=0$ implies $c_{0}<p-1$ [20, p. 5]. Write $n=c_{0}+p n_{0}$. Then $\gamma^{c_{0}} \zeta^{n_{0}}=\gamma^{n}-c_{1} \gamma^{n-p+1} \beta^{p-1}+\cdots$, and $N\left(\gamma^{c_{0}} \zeta^{n_{0}}\right)$ $=0$, as $\zeta \in \mathscr{A}^{k}$ and $c_{0}<p-1$, by (6.30). Thus $N \xi=0$ implies $N\left(\xi-\gamma^{c_{0}} \zeta^{n_{0}}\right)=0$; and degree $\left(\xi-\gamma^{c_{0}} \zeta^{n_{0}}\right)<$ degree $\xi$. By induction $\xi-\gamma^{c_{0}} \zeta^{n_{0}} \in \sum_{i=0}^{p-2} \gamma^{i} \mathscr{A}^{k}$. Hence so is $\xi$, since $c_{0}<p-1$. This proves ${ }_{N} \mathscr{A} \subseteq \sum_{i=0}^{p-2} \gamma^{i} \mathscr{A}^{k}$. By (6.30) the opposite inclusion holds as well. This proves (4).

$\gamma^{k-1}=\beta, \quad\left(\gamma^{2}\right)^{k-1}=2 \gamma \beta+\beta^{2}, \ldots$. This proves $\gamma^{i} \beta \in \mathscr{A}^{k-1}, 0 \leqq i<p-1$. As $\mathscr{A}^{k} \mathscr{A}^{k-1} \subseteq \mathscr{A}^{k-1}$, we see by (4) that $\mathscr{A}^{k-1} \supset \beta_{N} \mathscr{A}$. For the reverse inclusion, it suffices to show $\left(\gamma^{n}\right)^{k-1} \in \beta_{N}$ for all $n>0$. If $n<p$, this is easy by (6.30). If $n>p$, $\left(\gamma^{n}\right)^{k-1}=\left(\gamma^{n-p}\right)^{k-1} \zeta+\beta^{p-1}\left(\gamma^{n-p+1}\right)^{k-1}$, and we conclude by induction. This proves (5). Q.E.D.

$E_{2} \approx H^{*}\left(Z_{p}, \mathscr{A}\right)$ as bigraded rings, up to a sign (see $\left.\S 1\right)$. The multiplication in $H^{*}\left(Z_{p}, \mathscr{A}\right)$ is given as follows: [3, Chapter XII, §7]. Let $\alpha \in H^{2}\left(Z_{p}, Z\right)$ be the maximal generator corresponding to $A \rightarrow 1 / p$. Then $\alpha^{i}: \mathscr{A}^{k} / N \mathscr{A} \cong H^{2 i}\left(Z_{p}, \mathscr{A}\right)$. 
$\alpha^{i}:{ }_{N} \mathscr{A} / \mathscr{A}^{k-1} \cong H^{2 i-1}\left(Z_{p}, \mathscr{A}\right)$. If $\eta \in H^{*}\left(Z_{p}, \mathscr{A}\right), \xi \in H^{*}\left(Z_{p}, \mathscr{A}\right)$ are homogeneous cohomology classes represented by $a$ (resp. $b) \in \mathscr{A}$, then $\eta \xi$ is represented by

$$
\begin{aligned}
& a b \quad \text { if } \operatorname{deg} \eta \text { or } \operatorname{deg} \xi \text { is even, } \\
& \sum_{0 \leqq i<j<p} a^{k^{i}} b^{k^{j}} \text { if } \operatorname{deg} \eta \text { and } \operatorname{deg} \xi \text { are odd. }
\end{aligned}
$$

This shows that $\alpha: E_{2}^{i, j} \rightarrow E_{2}^{i+2, j}$ is iso for $i>0$. It is easy to check that $\zeta: E_{2}^{i, j}$ $\rightarrow E_{2}^{i, j+2 p}$ is iso for $i, j>0$. Let $\chi \in E_{2}^{1,3}$ correspond to $\mu \in \mathscr{A}^{3}, \nu_{i} \in E_{2}^{1,2 i}$ to $\gamma^{i}$, $1 \leqq i<p$, and $\sigma \in E_{2}^{1,2 p}$ to $\zeta$.

LEMMA 6.32. In $E_{2}$,

$$
\begin{aligned}
\beta \nu_{i} & =\beta \chi=\beta \sigma=0, \quad 1 \leqq i<p-1 . \\
\sigma^{2} & =\nu_{i}^{2}=\chi^{2}=\mu \chi=0 \\
\nu_{1} \chi & =\alpha \beta \mu \quad(p=3), \\
& =0 \quad p>3 .
\end{aligned}
$$

Proof. $\beta v_{i}$ corresponds to $\beta \gamma^{i} \in \mathscr{A}^{k-1}$ if $i<p-1$. Therefore $\beta v_{i}=0$. $\beta \chi$ corresponds to $\beta \mu \in \mathscr{A}^{k-1}$ implies $\beta \chi=0$. Likewise, $\beta \zeta \in \mathscr{A}^{k-1}$ implies $\beta \sigma=0$. This proves (1). We leave (2) as an exercise, and will prove (3).

Suppose $p>3$. Then as $\nu_{1}$ corresponds to $\gamma$, and $\chi$ to $\mu$, using the rule (6.31), $\nu_{1} \chi$ corresponds to $\sum_{0 \leqq i<j<p} \gamma^{k^{l}} \mu^{k^{l}}=-s_{2} \mu \beta . p>3$ implies $s_{2}=0, p=3$ implies $s_{2}=-1$. Thus $p>3$ implies $\nu_{1} \chi=0$. If $p=3$, since $\nu_{1} \chi \in E_{2}^{2,5}$, and $\alpha \beta \mu \in E_{2}^{2,5}$ also corresponds to $\mu \beta$, so $\nu_{1} \chi=\alpha \beta \mu$. Q.E.D.

Definition. If $u, v$ are quantities, we say $u \doteq v$ if and only if $u=e v, e \in Z_{p}^{*}$.

LEMMA 6.33. $d_{2}\left(\nu_{2}\right) \doteq \alpha \chi, d_{2}\left(\nu_{i}\right) \doteq \alpha \mu \nu_{i-2}, 3 \leqq i<p-2, d_{2}\left(\mu \nu_{i}\right)=0,1 \leqq i<p-1$.

Proof. We know that $\alpha_{2}=\alpha_{3}=p^{2}, \alpha_{4}=p^{4}$. This shows that $\alpha, \beta, \nu_{1}, \mu, \chi$ survive to $E_{\infty} . \alpha_{5}=p^{3}$ implies $\nu_{2}$ perishes. As $\alpha^{3}, \alpha^{2} \beta$ are linearly independent, $d_{2}\left(\nu_{2}\right)=d_{3}\left(\nu_{2}\right)$ $=0, d_{5}\left(\nu_{2}\right) \neq 0$, or $d_{2}\left(\nu_{2}\right)=0, d_{3}\left(\nu_{2}\right) \neq 0$ are not possible, implying $d_{2}\left(\nu_{2}\right) \doteq \alpha \chi$. We now proceed inductively, using (1) our knowledge of the $\alpha_{i},(2)$ the linear independence of the $\left\{\alpha^{k} \beta^{l}\right\}$ and $\left\{\alpha^{k} \beta^{l} \mu\right\} \cup\left\{\alpha^{k+l} \nu\right\}$, in certain ranges. We omit details. Done.

Let $E_{\infty}^{(n)}=E_{\infty}^{0, n} \oplus E_{\infty}^{1, n-1} \oplus \cdots \oplus E_{\infty}^{n, 0}$ in this order.

Corollary 6.34. $E_{3}=E_{\infty}$ in dimensions $\leqq 2 p$.

$$
\begin{aligned}
E_{\infty}^{(4)} & =Z \beta^{2} \oplus Z \chi \oplus Z \alpha \beta \oplus Z \alpha^{2} . \\
E_{\infty}^{(2 i)} & =Z \beta^{i}+Z \mu \nu_{i-2} \oplus Z \alpha \beta^{i-1} \oplus Z \alpha^{2} \beta^{i-2} \oplus \cdots \oplus Z \alpha^{i}, \quad 3 \leqq i \leqq p-1 . \\
E_{\infty}^{(2 i+1)} & =Z \beta^{i-1} \mu \oplus Z \alpha \beta^{i-2} \mu \oplus Z \alpha^{2} \beta^{i-3} \mu \oplus \cdots \oplus Z \alpha^{i-1} \mu \oplus Z \alpha^{i-1} \nu, \\
& 2 \leqq i \leqq p-2 . \\
E_{\infty}^{(2 p)} & =Z \zeta \oplus Z \beta^{p}+Z \mu \nu_{p-2} \oplus Z \alpha \mu \nu_{p-3} \oplus Z \alpha^{2} \beta^{p-2} \oplus Z \alpha^{3} \beta^{p-3} \oplus \cdots \oplus Z \alpha^{p} .
\end{aligned}
$$


Comparing with Theorem 6.26 and its lemmas, we see that $\chi_{1} \in H^{*}(G, Z)$ projects onto $\mu \nu_{i-1}, 2 \leqq i \leqq p-2$. Notation: $\pi\left(\chi_{i}\right) \doteq \mu \nu_{i-2}$. Also $\pi(\mu)=\mu, \pi(\nu) \doteq \nu_{1}$.

LEMMA 6.34. $d_{2}(\sigma) \doteq \alpha \mu v_{p-2}$.

Proof. As $\pi\left(\right.$ Cor $\left.\gamma^{p}\right) \doteq \mu v_{p-2}$ (easily checked), and $\alpha$ Cor $\gamma^{p}=0$, so $\alpha \mu v_{p-2}$ must be zero in $E_{\infty}$. But $\alpha \mu \nu_{p-2}$ is a universal cycle. Therefore it must be hit from behind. The only possibility is $d_{2}(\sigma) \doteq \alpha \mu \nu_{p-2}$. Q.E.D.

It is now easy to see that $E_{3}=E_{\infty}$. At this point we leave the second spectral sequence.

6.5. Final remark. Consider the three statements (a) $G \cong H$, (b) $Z G \cong Z H$ as rings, (c) $H^{*}(G, Z) \cong H^{*}(H, Z)$ as rings. Does (b) imply (a)? This is called the group ring problem, and is unsolved, although the answer is yes for special classes of groups. Using Yoneda's definition of group cohomology in terms of long exact sequences [see MacLane's Homology, Chapter III] one sees that $H^{*}(G, Z)$ (as ring) is determined by the augmented ring $Z G \stackrel{\varepsilon}{\rightarrow} Z$. Moreover, any ring isomorphism $\theta: Z G \stackrel{\cong}{\rightarrow} Z H$ may be modified so as to commute with augmentation. Thus,

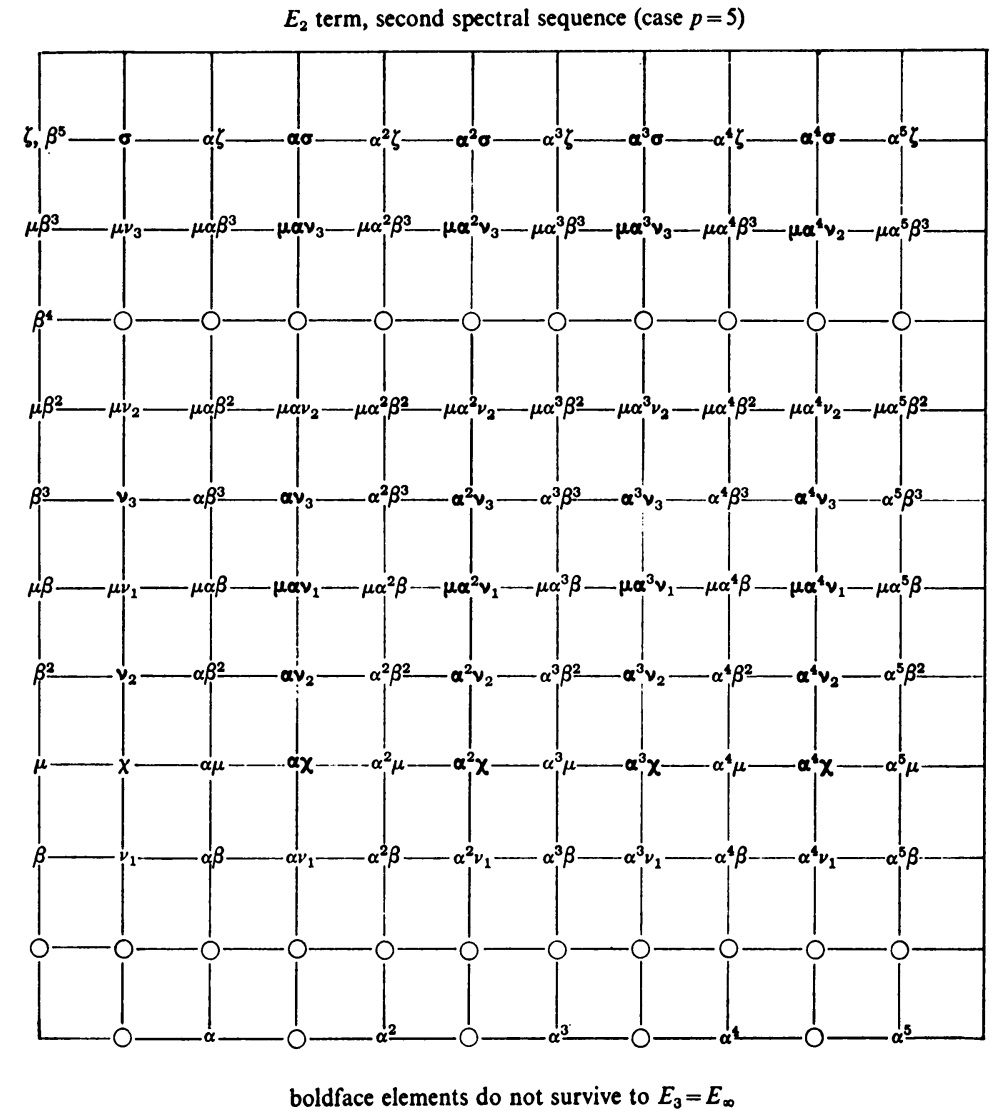


(b) implies (c). For non-p groups, (c) implies (a) is false, as shown by Alperin and Atiyah (unpublished) [essentially because $1 \rightarrow N \rightarrow G \rightarrow K \rightarrow 1$, exact, $(|N|,|K|)$ $=1$, implies $H^{*}(G, Z) \cong H^{*}(K, Z) \oplus H^{*}(N, Z)^{k}$, as rings]. For $p$-groups, (c) implies (a) may be true. Certainly our results show that (c) implies (a) for groups of order $p^{3}$; in fact, $H^{2}$ and $H^{3}$ suffice to determine $G$. Note also that groups of types I and II have the same character table [Burnside, Theory of groups, p. 319, Exercise 4], but different cohomology rings.

ACKNOWLEDGEMENTS. I wish to thank the following persons:

My thesis advisor, Professor Alex Heller, for his continued help and encouragement.

Professor Leonard Evens, who kindly showed me his results and conjectures concerning groups of type II, in the case $p=3$. He had calculated the multiplication

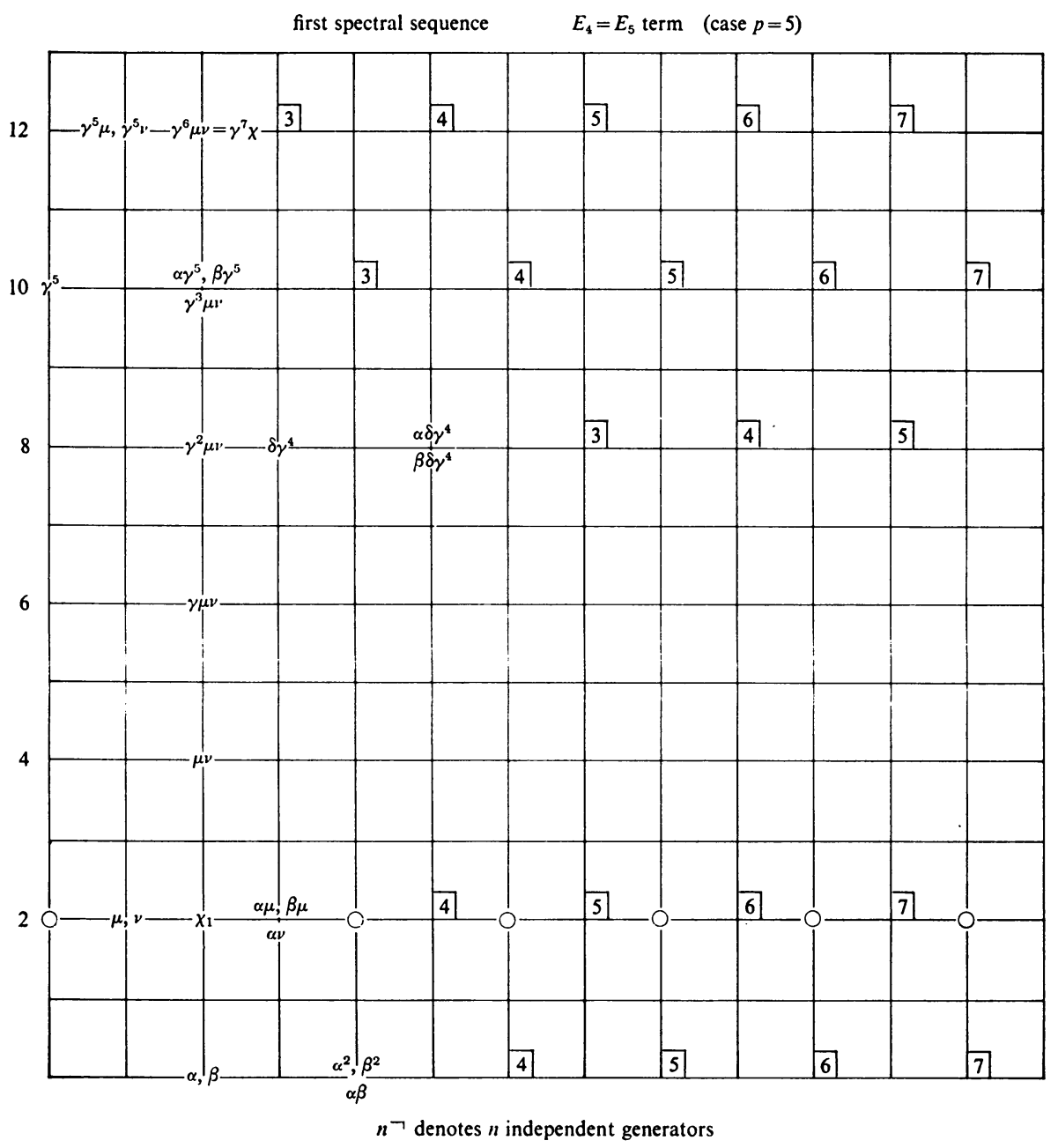


and various differentials in the $E_{2}$ terms of the first and second spectral sequences (see §6), and had correctly conjectured the relations in the cohomology ring.

Professor R. G. Swan, for helpful conversations. Also, Professor J. Alperin, Miss Tze-Nan Kuo, Professor Melvin Rothenberg, and Professor Norman Steenrod.

\section{BIBLIOGRAPHY}

1. M. F. Atiyah, Characters and cohomology of finite groups, Inst. Hautes Études Sci. Publ. Math. 9 (1961).

2. H. Cardenas, The cohomology algebra of $\Sigma_{p^{2}}$, coefficients $Z_{p}$, Thesis, Princeton Univ., Princeton, N. J., 1965.

3. H. Cartan and S. Eilenberg, Homological algebra, Princeton Univ. Press, Princeton, N. J., 1956.

4. L. Evens, The cohomology ring of a finite group, Trans. Amer. Math. Soc. 101 (1961), 224-239.

5. - A generalization of the transfer map in cohomology of groups, Trans. Amer. Math. Soc. 108 (1963), 54-65.

6. - On the Chern classes of representations of finite groups, Trans. Amer. Math. Soc. 115 (1965), 180-193.

7. P. J. Hilton and D. Rees, Natural maps of extension functors and a theorem of R. G. Swan, Proc. Cambridge Philos. Soc. 57 (1961), 489-502.

8. G. P. Hochschild and J.-P. Serre, Cohomology of group extensions, Trans. Amer. Math. Soc. 74 (1953), 110-135.

9. M. Nakaoka, Homology of the infinite symmetric groups, Ann. of Math. 73 (1961), 229-257.

10. - Note on cohomology algebras of symmetric groups, J. Math. Osaka City Univ. 13 (1962), 45-55.

11. N. Steenrod and D. B. A. Epstein, Cohomology operations, Princeton Univ. Press, Princeton, N. J., 1962.

12. R. G. Swan, A new method in fixed point theory, Comment Math. Helv. 34 (1960).

13. - The p-period of a finite group, Illinois J. Math. 4 (1960), 341-346.

14. - Periodic resolutions for finite groups, Ann. of Math. 72 (1960), 267-291.

15. J. Tate, Homology of Noetherian rings and local rings, Illinois J. Math. 1 (1957), 14-27.

16. C. T. C. Wall, Resolutions for extensions of groups, Proc. Cambridge Philos. Soc. 57 (1961), 251-255.

17. H. Zassenhaus, The theory of groups, Chelsea, New York, 1949.

\section{UNIVERSITY OF CALIFORNia, Berkeley, CALIForNia}

